\title{
Inheritance of Genetic variability, Combining Ability and Heterosis for Yellow Mosaic Virus Disease Resistance and Yield Improvement in Blackgram [Vigna mungo (L.) Hepper]
}

\author{
R. Suguna, P. Savitha* and C.R. Ananda Kumar \\ Department of Plant Breeding and Genetics, Tamil Nadu Agricultural University, \\ Coimbatore, Tamil Nadu, India \\ *Corresponding author
}

\begin{tabular}{|c|c|}
\hline & A B S T R A C T \\
\hline & \multirow{5}{*}{$\begin{array}{l}\text { Pulses are the second most important group of crops grown worldwide. Among pulses, } \\
\text { black gram (Vigna mungo L. Hepper) occupies a prominent place in India. Black gram } \\
\text { grain contains about } 24 \% \text { protein, } 60 \% \text { carbohydrates, } 1.3 \% \text { fat with desirable amount of } \\
\text { minerals like calcium, phosphorus, iron and certain vitamins. Yellow mosaic virus is one } \\
\text { of the most important constraints for blackgram production. To identify genetic sources of } \\
\text { resistance to yellow mosaic virus (YMV) in blackgram, the genetic variability is lost and it } \\
\text { is this genetic potential for high yield needs to be regenerated. Four parents viz., Vamban } \\
4 \text {, Vamban } 2 \text {, LBG } 17 \text { and CO } 5 \text { and their } 12 \text { hybrids, obtained through full diallel mating } \\
\text { design were evaluated for important quantitative traits during Rabi, 2010-2011 for YMV } \\
\text { and improvement of yield. Genetic variability, the PCV value was found higher in all the } \\
\text { characters studied except days to } 50 \text { percent flowering, days to maturity and number of } \\
\text { seeds per pod than the GCV. Based on per se performance, gca effects and sca effects, CO } \\
5 \text { x VBN } 2 \text { cross combination was found to be superior which combine yield and quality } \\
\text { characters and these hybrid can be utilized for recombination breeding. Based on per se } \\
\text { performance, sca effects and standard heterosis, two cross combinations viz., LBG } 17 \times \\
\text { CO } 5 \text { and VBN } 2 \text { x LBG } 17 \text { was found to be superior which combine yield and quality } \\
\text { characters and these hybrids can be utilized for heterosis breeding. Investigation on the } \\
\text { magnitude of heterosis helps to identify promising hybrid combination and also possible to } \\
\text { exploit to new recombinant type for yield and attributing traits from segregants. }\end{array}$} \\
\hline & \\
\hline Article Info & \\
\hline $\begin{array}{l}\text { Accepted: } \\
\text { 17 September } 2017 \\
\text { Available Online: } \\
10 \text { November } 2017\end{array}$ & \\
\hline & \\
\hline
\end{tabular}

\section{Introduction}

Pulses are the second most important group of crops grown worldwide. Indian has the largest area of about 34 per cent and total production of about 26 per cent of pulses globally. The Mungbean Yellow Mosaic Virus disease (MYMV) is a highly devastating disease in tropical and sub-tropical Asia. MYMV belongs to genus Begomovirus of the family Geminiviridae (Bos, 1999). The virus has geminate particle morphology $(20 \times 30 \mathrm{~nm})$ and the coat protein encapsulates spherical, single stranded DNA genome of approximately $2.8 \mathrm{~Kb}$ (Hull, 2004). The first symptom appears on young leaves as yellow specks or spots. The leaf emerging from the apex shows bright yellow patches interspersed with green areas. In severe cases there is a complete yellowing of the leaves and infected 
plants stunted. They bear few flowers and pods and mature late. The yield losses in naturally infected susceptible cultivars varied with time of infection (Singh et al., 1982). Early infected plants had more severe symptoms than late infected ones. Chlorosis, stunting and reduced branching contributed to yield loss. The concept of combining ability analysis helps in selection of superior parents (general combining ability) as well as crosses (specific combining ability) when considered along with the mean performances. It also tells about the nature of gene action involved and thus helps in framing a suitable breeding scheme for the amelioration of the characters under consideration. General combining ability is used to designated those crosses in which certain combinations do relatively better or worse than would expected on the basis of the average performance of the lines involved. Different mating systems have been developed for estimating the combining ability and to derive the gene action in the inheritance of polygenic characters. This technique has been extensively used in almost all the major field crops to estimate GCA and SCA variances and effects and to understand the nature of gene action involved in the expression of various quantitative traits. The breeders need sound information on variability consisting of phenotypic and genotypic variance to obtain better results for selecting superior genotypes. Heritability refers to 'the extent of transmission of variation for any trait to the progeny'. Estimate of heritability helps in discriminating the variance in a population into the genotypic component and environment interaction component and explain the relative importance of environment effect and inheritance levels for the variation in population. Genetic advance is a measure of the gain for the character that could be achieved by further selection. Heritability along with genetic advance estimates helps in programming the breeding programme to obtain best results of genetic gain for any economic trait. Heterosis refers to the increased or decreased vigour of $F_{1}$ hybrid over its parents. The term heterosis was coined by Shull (1914). According to him, the term heterosis refers to the increased vigour, growth, yield or functions of hybrid over the parents those results from crossing genetically diverse individuals. The possibility of commercial exploitation of hybrid vigour in crops like green gram and black gram depends upon the substantial heterosis for YMV and seed yield coupled with economically viable method of producing hybrid seeds.

\section{Materials and Methods}

The present investigation was conducted at the Agricultural College and Research Institute, Madurai during 2010-2011 at the experimental farm in the Department of Plant Breeding and Genetics. Four varieties of blackgram obtained from National Pulses Research Centre, Vamban, Tamil Nadu. Among the parents, four genotypes viz., Vamban 4, Vamban 2, LBG 17 and CO 5 were used as the materials of the present study. Twelve hybrids were raised during Rabi, 2011 in ridges of three meter length with an inter row spacing of $40 \mathrm{~cm}$ and intrarow spacing of $20 \mathrm{~cm}$. The hybrids were raised in a Randomized Block Design with three replications. For estimating heterosis, the parents were also raised in adjacent plot with above mentioned spacing in three replications. The recommended agronomic and plant protection practices were followed to maintain healthy stand of the plants. The Yellow Mosaic Virus Disease (YMV) incidence was recorded on all the plants based on the visual scores on $50^{\text {th }}$ day while the susceptible check C0 5 recorded scale 6.9. The classification was made into scales $1-9$ as follows based on the scale adopted by Singh et al., (1988). Combining ability 
analysis of cultivars is thus important to exploit the relevant type of gene action for a breeding programme. Combining ability estimates can be used to evaluate the number of promising lines in $F_{1}$ and $F_{2}$ generations, which is quite helpful in selecting the potential parents for hybridization. Combining ability study is useful in classifying the parental lines in terms of their hybrid performance (Dhillon, 1975). It also helps in identifying the parents suitable for hybridization programme and deciding suitable breeding methodology (Table 12).

\section{Results and Discussion}

Success in any breeding programme largely depends on the knowledge of the genetic architecture of the population handled by the breeder. The estimate of components of variance provides an idea about additive and non-additive (dominant) types of gene action (Baker, 1978). Panse (1942) suggested that if additive variance is greater than non-additive variance, the chance of fixing superior genotypes in the early segregating generations would be greater. Recent developments in the biometrical methods have led to the formulation of a number of statistical procedures for the genetic analysis of quantitative characters. Diallel analysis is one among them, which provides information on additive and non-additive gene action, inferred from Diallel analysis. The magnitude of $\mathrm{H}_{1}$ variances was higher than $\mathrm{D}$ variances for all the traits. The number of days to 50 percent flowering ranged between 34.33 to 37.33 days. The grand mean for this trait was 35.83days. Among the parent $\mathrm{P}_{2}$ was the earliest in flowering and for this trait all other parents recorded non-significant value with that of the respective mean. Days to 50 per cent flowering among the hybrids varied from $34.66\left(\mathrm{P}_{2} \times \mathrm{P}_{3}\right)$ to 37.00 days $\left(\mathrm{P}_{1} \times \mathrm{P}_{4}\right.$ and $\mathrm{P}_{4} \mathrm{x}$ $\mathrm{P}_{1}$ ). The grand mean for this trait was 35.77 days. Out of this 12 hybrids, only one hybrid namely $\mathrm{P}_{2} \times \mathrm{P}_{3}$ recorded significantly early in flowering than the grand mean. The gca effects ranged from (-0.729) $\mathrm{P}_{2}$ to $(0.771) \mathrm{P}_{4}$. Significant negative values of $\mathrm{gca}$ was obtained by $\mathrm{P}_{2}$ and in the parent $\mathrm{P}_{4}$ showed significantly positive $\mathrm{gca}$ effects for this trait. The sca effects for days to 50 per cent flowering ranged from $-0.436\left(\mathrm{P}_{4} \mathrm{x}_{2} \mathrm{P}_{2}\right)$ to $0.649\left(\mathrm{P}_{2} \times \mathrm{P}_{1}\right)$. Out of 12 crosses, three combinations viz., $\mathrm{P}_{2} \times \mathrm{P}_{1}$ alone registered positively significant $s c a$ effects (Fig. 3). The hybrids $\mathrm{P}_{3} \times \mathrm{P}_{4}$ and $\mathrm{P}_{4} \times \mathrm{P}_{2}$ had exhibited negative significant $s c a$ effect for this trait. In combining ability analysis, the estimate of the additive genetic variance (D) was found higher than the dominant genetic variance $\left(\mathrm{H}_{1}\right)$. It implied the preponderance of additive gene action for days to 50 per cent flowering. Srividhya et al., (2005) and Barad et al., (2008) obtained similar gene action in their studies, whereas preponderance of nonadditive gene action was reported by Vaithiyalingam (2002), Pooran Chand and Raghunadha Rao (2002), Anbumalarmathi et al., (2004), Abdul Ghaffor and Zahoor Ahmad (2005), Bhagirath et al., 2013, Yashpal et al., 2015, Kachave et al., 2015 and Thamodharn et al., 2016 for this trait. The relative heterosis for this trait ranged from $0.47\left(\mathrm{P}_{3} \times \mathrm{P}_{2}\right)$ to 1.87 percent $\left(\mathrm{P}_{4} \times \mathrm{P}_{2}\right)$. Out of 12 hybrids, all hybrids exhibited nonsignificant relative heterosis. Maximum heterobeltiosis was observed within range $4.46\left(\mathrm{P}_{2} \times \mathrm{P}_{4}\right)$ to 0.00 per cent $\left(\mathrm{P}_{3} \times \mathrm{P}_{1}\right.$ and $\mathrm{P}_{4}$

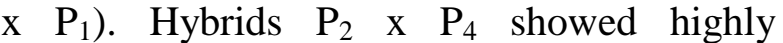
significant negative heterobeltiosis and $\mathrm{P}_{2} \mathrm{X}$ $\mathrm{P}_{3}, \mathrm{P}_{4} \times \mathrm{P}_{3}$ and $\mathrm{P}_{3} \times \mathrm{P}_{4}$ showed significant negative heterobeltiosis. The heterosis percentage over standard variety varied from $3.57\left(\mathrm{P}_{3} \times \mathrm{P}_{1}\right.$ and $\left.\mathrm{P}_{3} \times \mathrm{P}_{4}\right)$ to -7.14 percent $\left(\mathrm{P}_{2}\right.$ $\mathrm{x} \mathrm{P}_{3}$ ). In this trait seven crosses $\mathrm{P}_{2} \times \mathrm{P}_{3}, \mathrm{P}_{1} \mathrm{x}$ $\mathrm{P}_{2}, \mathrm{P}_{2} \times \mathrm{P}_{1}, \mathrm{P}_{3} \times \mathrm{P}_{2}, \mathrm{P}_{1} \times \mathrm{P}_{3}, \mathrm{P}_{2} \times \mathrm{P}_{4}$ and $\mathrm{P}_{4} \times \mathrm{P}_{3}$ showed highly significant negative standard heterosis and the hybrids $\mathrm{P}_{3} \times \mathrm{P}_{1}$ and $\mathrm{P}_{3} \times \mathrm{P}_{4}$ recorded significant negative heterosis (Table 5) (Figs. 4, 5, 6). 
The grand mean for days to maturity was (62.33) $\mathrm{P}_{2} \times \mathrm{P}_{1}$ to (72.33 days) $\mathrm{P}_{4} \times \mathrm{P}_{1}$ and with this trait only one cross $\mathrm{P}_{2} \times \mathrm{P}_{1}$ showed significantly early in maturity than their grand mean (66.14 days) (Table 1). For days to maturity, the lowest value of gca was showed by the parent $(-3.042)$ and the highest value by (2.208). Significantly negative $\mathrm{gca}$ effects were recorded by $\mathrm{P}_{2}$ and the parent $\mathrm{P}_{4}$ and $\mathrm{P}_{1}$ registered significantly positive $g c a$ effects for this trait (Table 2). Among twelve crosses, five showed significant and positive sca effects while six showed significantly negative sca effects (Table 3$)$. The cross, $\left(\mathrm{P}_{3}\right.$ $\left.x \mathrm{P}_{2}\right),\left(\mathrm{P}_{4} \times \mathrm{P}_{3}\right)$ exhibited the lowest sca effect $(-0.83)$ whereas $\left(\mathrm{P}_{2} \times \mathrm{P}_{3}\right)$ showed the highest sca effect (3.16). In Diallel analysis, dominant genetic variance $\left(\mathrm{H}_{1}\right)$ was found lesser than that of additive genetic variance (D) indicating the additive gene action for this trait. Aher et al., (2001) and Abdul Ghaffor and Zahoor Ahmad (2003) noticed the additive gene action for days to maturity. Some authors namely, Abdul Ghaffor and Zahoor Ahmed (2003 and 2005), Jayapradha et al., (2005), Srividhya et al., (2005) and Barad et al., (2008), Vijay kumar et al., 2014, Thamodharn et al., 2016 reported dominant gene action for this trait. The relative heterosis ranged from $-3.70\left(\mathrm{P}_{3} \times \mathrm{P}_{4}\right)$ to 10.80 percent $\left(\mathrm{P}_{3} \times \mathrm{P}_{2}\right)$ and eight hybrids namely $\mathrm{P}_{3}$ x $\mathrm{P}_{2}, \mathrm{P}_{1} \times \mathrm{P}_{2}, \mathrm{P}_{2} \times \mathrm{P}_{3}, \mathrm{P}_{4} \times \mathrm{P}_{1}, \mathrm{P}_{2} \times \mathrm{P}_{4}, \mathrm{P}_{4} \times \mathrm{P}_{2}$ and $\mathrm{P}_{2} \quad \mathrm{x} \quad \mathrm{P}_{1}$ registered highly significant positive relative heterosis and $\mathrm{P}_{3} \times \mathrm{P}_{4}$ alone showed highly significant negative heterosis. Heterobeltiosis ranged between $-3.38\left(\mathrm{P}_{4} \mathrm{x}\right.$ $\left.\mathrm{P}_{3}\right)$ and 4.83 percent $\left(\mathrm{P}_{4} \times \mathrm{P}_{1}\right)$. Out of 12 hybrids, a total of five crosses $\mathrm{P}_{3} \times \mathrm{P}_{4}, \mathrm{P}_{2} \times \mathrm{P}_{1}$, $\mathrm{P}_{2} \times \mathrm{P}_{4}, \mathrm{P}_{4} \times \mathrm{P}_{2}$ and $\mathrm{P}_{4} \times \mathrm{P}_{3}$ showed highly significant negative heterobeltiosis and $\mathrm{P}_{4} \times \mathrm{P}_{1}$ alone exhibited highly significant and positive heterobeltiosis. Standard heterosis varied from -3.38 $\left(\mathrm{P}_{3} \times \mathrm{P}_{2}\right.$ and $\left.\mathrm{P}_{4} \times \mathrm{P}_{3}\right)$ to 4.83 percent $\left(\mathrm{P}_{4} \times \mathrm{P}_{1}\right)$. The crosses namely $\mathrm{P}_{2} \times \mathrm{P}_{1}$, $\mathrm{P}_{1} \times \mathrm{P}_{3}, \mathrm{P}_{2} \times \mathrm{P}_{3}, \mathrm{P}_{3} \times \mathrm{P}_{4}, \mathrm{P}_{1} \times \mathrm{P}_{2}, \mathrm{P}_{3} \times \mathrm{P}_{1}, \mathrm{P}_{2} \times$ $\mathrm{P}_{4}, \mathrm{P}_{4} \times \mathrm{P}_{2}, \mathrm{P}_{3} \times \mathrm{P}_{2}$ and $\mathrm{P}_{4} \times \mathrm{P}_{3}$ showed highly significant negative standard heterosis and $\mathrm{P}_{4}$ $\mathrm{x} \mathrm{P}_{1}$ alone recorded significantly positive standard heterosis (Table 5).

The minimum and maximum plant height was recorded in the hybrid (30.75) $\mathrm{P}_{2} \times \mathrm{P}_{1}$ to $(45.00 \mathrm{~cm}) \mathrm{P}_{4} \times \mathrm{P}_{3}$. The crosses $\mathrm{P}_{4} \times \mathrm{P}_{3}, \mathrm{P}_{3} \times$ $\mathrm{P}_{2}, \mathrm{P}_{3} \times \mathrm{P}_{1}, \mathrm{P}_{2} \times \mathrm{P}_{4}, \mathrm{P}_{4} \times \mathrm{P}_{1}, \mathrm{P}_{4} \times \mathrm{P}_{2}, \mathrm{P}_{1} \times \mathrm{P}_{4}$, $\mathrm{P}_{1} \times \mathrm{P}_{2}, \mathrm{P}_{2} \times \mathrm{P}_{3}$ and $\mathrm{P}_{2} \times \mathrm{P}_{1}$ recorded significantly higher plant height compared to their grand mean $(39.43 \mathrm{~cm})$. The $\mathrm{gca}$ effect for plant height varied from $\mathrm{P}_{1}(-2.193)$ to $\mathrm{P}_{3}$ (2.895). However, in general, it was observed that all of the parents showed significant $g c a$ effects for this trait. Significantly negative gca effects were observed in $\mathrm{P}_{1}$ and $\mathrm{P}_{2}$. The parent $\mathrm{P}_{3}$ and $\mathrm{P}_{4}$ recorded significantly positive gca effects for this trait. For the trait plant height, the sca values fell between -1.29 $\left(\mathrm{P}_{3} \times \mathrm{P}_{1}\right)$ to $3.71\left(\mathrm{P}_{1} \times \mathrm{P}_{3}\right)$. Of these, seven hybrids $\mathrm{P}_{1} \times \mathrm{P}_{3}, \mathrm{P}_{2} \times \mathrm{P}_{4}, \mathrm{P}_{4} \times \mathrm{P}_{2}, \mathrm{P}_{2} \times \mathrm{P}_{3}, \mathrm{P}_{2} \times$ $\mathrm{P}_{1}, \mathrm{P}_{1} \times \mathrm{P}_{2}$, and $\mathrm{P}_{1} \times \mathrm{P}_{4}$ showed significant and positive sca effects. Four crosses exhibited significantly negative effects for this trait. The estimate of dominance genetic variance $\left(\mathrm{H}_{1}\right)$ was greater than additive genetic variance (D) for plant height. This inferred that nonadditive gene action governed this trait. Manivannan (2002), Vaithiyalingam et al., (2002), Anbumalarmathi et al., (2004), Srividhya et al., (2005), Barad et al., (2008), Supriyo Chakraborty et al., (2010, Vijay kumar et al., 2014, Kachave et al., 2015 Thamodharn et al., 2016 observed predominance of the non-additive gene action in controlling this trait. The relative heterosis for this trait ranged from $2.98\left(\mathrm{P}_{1} \times \mathrm{P}_{4}\right)$ to 37.51 percent $\left(\mathrm{P}_{3} \times \mathrm{P}_{2}\right)$. A total of 12 crosses registered highly significant positive relative heterosis. The heterosis percentage over better parent ranged from $-9.45\left(\mathrm{P}_{1} \times \mathrm{P}_{4}\right)$ to 21.12 percent $\left(\mathrm{P}_{4} \times \mathrm{P}_{3}\right)$ and the hybrids such as $\mathrm{P}_{4} \mathrm{X}$ $\mathrm{P}_{3}, \mathrm{P}_{3} \times \mathrm{P}_{2}, \mathrm{P}_{1} \times \mathrm{P}_{2}, \mathrm{P}_{3} \times \mathrm{P}_{1}, \mathrm{P}_{2} \times \mathrm{P}_{4}, \mathrm{P}_{4} \times \mathrm{P}_{1}$, $\mathrm{P}_{2} \times \mathrm{P}_{1}, \mathrm{P}_{1} \times \mathrm{P}_{3}, \mathrm{P}_{3} \times \mathrm{P}_{4}$ and $\mathrm{P}_{4} \times \mathrm{P}_{2}$ showed highly significant positive heterobeltiosis and only two cross $\mathrm{P}_{2} \times \mathrm{P}_{3}$ and $\mathrm{P}_{1} \times \mathrm{P}_{4}$ recorded 
highly significant negative heterosis over better parent. The minimum and maximum standard heterosis was observed in-10.03 $\left(\mathrm{P}_{1} \mathrm{X}\right.$ $\left.\mathrm{P}_{4}\right)$ and 21.12 percent $\left(\mathrm{P}_{4} \times \mathrm{P}_{3}\right)$. Seven hybrids exhibited highly significant positive standard heterosis and $\mathrm{P}_{4} \times \mathrm{P}_{2}$ alone showed significant positive heterosis. Four crosses showed highly significant negative standard heterosis (Table 5).

The number of branches per plant ranged from (2.52) $\mathrm{P}_{2}$ to (3.21) $\mathrm{P}_{4}$. The grand mean for this trait was 3.31. The parent $\mathrm{P}_{2}$ alone registered significantly positive mean value for this trait. The variation for this trait ranged from 3.00 to 4.19. Out of 12 hybrids, two $\begin{array}{llllllllll}\text { hybrids } & \mathrm{P}_{2} & \mathrm{x} & \mathrm{P}_{3} & \text { and } & \mathrm{P}_{3} & \mathrm{x} & \mathrm{P}_{4} & \text { recorded }\end{array}$ significantly more number of branches with that of the grand mean (3.69). Among the parents, gca effects for number of branches varied from -0.215 to 0.218 . Positive and significant gca effects were observed in $\mathrm{P}_{4}$. The gca effects were significant and negative for the parent $\mathrm{P}_{2}$. The hybrids had the lowest and the highest sca effects of $\mathrm{P}_{4} \times \mathrm{P}_{1}(-0.317)$ and $\mathrm{P}_{3} \times \mathrm{P}_{4}(0.40)$ respectively. The sca effects were significant and positive in the hybrids namely $\mathrm{P}_{3} \times \mathrm{P}_{4}, \mathrm{P}_{2} \times \mathrm{P}_{4}, \mathrm{P}_{1} \times \mathrm{P}_{3}, \mathrm{P}_{1} \times$ $\mathrm{P}_{2}$ and $\mathrm{P}_{4} \times \mathrm{P}_{2}$ and three cross $\mathrm{P}_{1} \times \mathrm{P}_{4}, \mathrm{P}_{4} \times \mathrm{P}_{1}$ and $\mathrm{P}_{3} \times \mathrm{P}_{2}$ showed significant and negative $s c a$ effects for the trait number of branches. The values of dominant genetic variance $\left(\mathrm{H}_{1}\right)$ exceeded the values of additive genetic variance (D) in combining ability analysis, thus exhibiting the presence of non-additive gene action for this trait. This was in conformity with earlier findings of Abdul Ghaffor and Zahoor Ahmed (2005) and Kachave et al., 2015. The preponderance of additive gene action was confirmed by Khattak et al., (2001), Anbumalarmathi et al., (2004) and Vijay kumar et al., 2014 for number of branches per plant. The hybrids expressed a range of relative heterosis from $24.89\left(\mathrm{P}_{1} \times \mathrm{P}_{3}\right)$ to 37.49 percent $\left(\mathrm{P}_{4} \times \mathrm{P}_{3}\right)$ and the crosses showing highly significant and positive heterosis were $\mathrm{P}_{4} \times \mathrm{P}_{3}, \mathrm{P}_{4} \times \mathrm{P}_{1}, \mathrm{P}_{3} \times \mathrm{P}_{4}, \mathrm{P}_{1} \times$ $\mathrm{P}_{2}, \mathrm{P}_{3} \times \mathrm{P}_{1}, \mathrm{P}_{2} \times \mathrm{P}_{4}, \mathrm{P}_{4} \times \mathrm{P}_{2}, \mathrm{P}_{3} \times \mathrm{P}_{2}$ and $\mathrm{P}_{1} \times$ $\mathrm{P}_{3}$. Heterobeltiosis ranged from $19.00\left(\mathrm{P}_{4} \mathrm{X}\right.$ $\left.\mathrm{P}_{2}\right)$ to 36.11 percent $\left(\mathrm{P}_{4} \times \mathrm{P}_{3}\right)$. Hybrids such as $\mathrm{P}_{4} \times \mathrm{P}_{3}, \mathrm{P}_{4} \times \mathrm{P}_{1}, \mathrm{P}_{3} \times \mathrm{P}_{4}, \mathrm{P}_{3} \times \mathrm{P}_{1}, \mathrm{P}_{2} \times \mathrm{P}_{4}, \mathrm{P}_{1} \times$ $\mathrm{P}_{3}, \mathrm{P}_{1} \times \mathrm{P}_{2}$ and $\mathrm{P}_{4} \times \mathrm{P}_{2}$ recorded highly significant positive heterobeltiosis. The heterosis percentage over the standard variety varied from $19.17\left(\mathrm{P}_{3} \times \mathrm{P}_{1}\right)$ to 30.47 percent $\left(\mathrm{P}_{3} \times \mathrm{P}_{4}\right)$. Hybrids namely $\mathrm{P}_{3} \times \mathrm{P}_{4}, \mathrm{P}_{4} \times \mathrm{P}_{3}, \mathrm{P}_{2} \times$ $\mathrm{P}_{4}, \mathrm{P}_{4} \times \mathrm{P}_{1}$ and $\mathrm{P}_{3} \times \mathrm{P}_{1}$ showed highly significant positive standard heterosis (Table 5).

The mean value of this Number of clusters per plant ranged from 12.39 to 16.98 with a grand mean 14.76. The parents $\mathrm{P}_{2}$ and $\mathrm{P}_{4}$ recorded significantly superior mean values than the grand mean. The mean values of number of clusters per plant among the hybrids range from $16.50\left(\mathrm{P}_{3} \times \mathrm{P}_{1}\right)$ to 23.50 $\left(\mathrm{P}_{1} \times \mathrm{P}_{2}\right)$. The hybrids $\mathrm{P}_{1} \times \mathrm{P}_{2}, \mathrm{P}_{3} \times \mathrm{P}_{4}, \mathrm{P}_{2} \times \mathrm{P}_{3}$, $\mathrm{P}_{4} \times \mathrm{P}_{3}, \mathrm{P}_{1} \times \mathrm{P}_{4}, \mathrm{P}_{2} \times \mathrm{P}_{4}, \mathrm{P}_{1} \times \mathrm{P}_{3}, \mathrm{P}_{3} \times \mathrm{P}_{2}, \mathrm{P}_{4} \times$ $\mathrm{P}_{1}, \mathrm{P}_{2} \times \mathrm{P}_{1}$ and $\mathrm{P}_{3} \times \mathrm{P}_{1}$ recorded significantly more number of clusters with that of the mean (19.88). The gca effect observed for this trait ranged from $-0.556\left(\mathrm{P}_{1}\right.$ and $\left.\mathrm{P}_{2}\right)$ to $0.880\left(\mathrm{P}_{4}\right)$. Significant and positive effects were noticed in $\mathrm{P}_{4}(0.880)$ and $\mathrm{P}_{3}(0.232)$. Significant and negative effects were observed in $\mathrm{P}_{1}$ and $\mathrm{P}_{2}$ (0.556). The lowest value of sca effect was shown by $\mathrm{P}_{1} \times \mathrm{P}_{3}(-0.52)$ and $\mathrm{P}_{3} \times \mathrm{P}_{4}$ the highest by (2.54). The hybrids with significant and positive sca effects were $\mathrm{P}_{3} \times \mathrm{P}_{4}, \mathrm{P}_{2} \times \mathrm{P}_{4}$, $\mathrm{P}_{2} \times \mathrm{P}_{1}, \mathrm{P}_{3} \times \mathrm{P}_{1}, \mathrm{P}_{4} \times \mathrm{P}_{3}$ and $\mathrm{P}_{1} \times \mathrm{P}_{4}$. Five registered negatively significant sca effects. Higher estimates of dominant genetic variance $\left(\mathrm{H}_{1}\right)$ than additive genetic variance (D) indicated the presence of dominant gene action for this trait. Singh and Dikshit (2003), Srividhya et al., (2005), Barad et al., (2008), Thamodharn et al., 2016 found similar type of gene action controlling this trait whereas the predominance of additive and non-additive type of gene action was reported by some 
workers in earlier findings viz., Jahagirdar (2001) Vijay kumar et al., 2014 and Tantasawat et al., 2015. The relative heterosis varied from $11.76\left(\mathrm{P}_{4} \times \mathrm{P}_{1}\right)$ to 78.08 percent $\left(\begin{array}{lll}\mathrm{P}_{1} & \mathrm{x} & \mathrm{P}_{2}\end{array}\right)$. Out of 12 hybrids, all hybrids recorded highly significant positive relative heterosis. The minimum and maximum heterobeltiosis were observed in $\mathrm{P}_{3} \times \mathrm{P}_{1}$ (10.00) and $\mathrm{P}_{1} \times \mathrm{P}_{2}$ (67.86 percent) with 11 hybrids showing highly significant positive heterobeltiosis. Standard heterosis varied between $7.22\left(\mathrm{P}_{3} \times \mathrm{P}_{2}\right)$ to 38.40 percent $\left(\mathrm{P}_{1} \mathrm{x}\right.$ $\mathrm{P}_{2}$ ) with nine crosses showed highly significant positive heterosis such as $\mathrm{P}_{1} \times \mathrm{P}_{2}$, $\mathrm{P}_{3} \times \mathrm{P}_{4}, \mathrm{P}_{2} \times \mathrm{P}_{3}, \mathrm{P}_{4} \times \mathrm{P}_{3}, \mathrm{P}_{1} \times \mathrm{P}_{4}, \mathrm{P}_{1} \times \mathrm{P}_{3}, \mathrm{P}_{2} \times \mathrm{P}_{4}$, $\mathrm{P}_{4} \times \mathrm{P}_{2}$ and $\mathrm{P}_{3} \times \mathrm{P}_{2}$ (Table 6).

Pod length of parents varied from 3.96 to 4.73 $\mathrm{cm}$. The grand mean for this trait was $4.50 \mathrm{~cm}$. Among the parents $\mathrm{P}_{2}$ alone produced significant mean value than the grand mean. Among the hybrids the lowest and the highest pod length was observed in $\mathrm{P}_{2} \times \mathrm{P}_{1}$ (4.50) to $\mathrm{P}_{3} \times \mathrm{P}_{1}(5.53 \mathrm{~cm})$ and out of this 12 hybrids, four hybrids $\mathrm{P}_{3} \times \mathrm{P}_{1}, \mathrm{P}_{4} \times \mathrm{P}_{3}, \mathrm{P}_{1} \times \mathrm{P}_{2}$ and $\mathrm{P}_{2} \times$ $\mathrm{P}_{1}$ recorded significantly higher pod length than that of the mean $(5.13 \mathrm{~cm})$. Among the parents, the $g c a$ values ranged from $\mathrm{P}_{2}$ ($0.253)$ to $P_{3}(0.192)$. The parent $P_{3}$ had significantly positive $g c a$ effects and $\mathrm{P}_{2}$ recorded significantly negative $\mathrm{gca}$ effects for this trait. The sca effects for pod length ranged from $\mathrm{P}_{4} \times \mathrm{P}_{1}(-0.10)$ to $\mathrm{P}_{2} \times \mathrm{P}_{4}(0.31)$. The four crosses $\mathrm{P}_{2} \times \mathrm{P}_{4}, \mathrm{P}_{1} \times \mathrm{P}_{3}, \mathrm{P}_{2} \times \mathrm{P}_{1}$, and $\mathrm{P}_{3} \times \mathrm{P}_{4}$ showed significant and positive sca effects and three crosses $\mathrm{P}_{2} \times \mathrm{P}_{3}, \mathrm{P}_{1} \times \mathrm{P}_{4}$ and $\mathrm{P}_{4} \times \mathrm{P}_{1}$ exhibited significantly negative sca effects for this trait. In combining ability analysis, the estimate of the additive genetic variance (D) was lesser than the dominant genetic variance $\left(\mathrm{H}_{1}\right)$. It indicated the preponderance of dominant gene action. Similar result was reported by Anbumalarmathi et al., (2004) Barad et al., (2008) and Baradhan and Thangavel (2011). Additive gene action was predominant in pod length and it was suggested by Srividhya $e t$ al., (2005), Saif Ullah Ajmal et al., (2007), Vijay kumar et al., 2014 and Yashpal et al., 2015. The relative heterosis for this trait ranged from 6.00 percent $\left(\mathrm{P}_{4} \times \mathrm{P}_{1}\right)$ to 19.53 percent $\left(\mathrm{P}_{2} \times \mathrm{P}_{3}\right)$. Ten hybrids recorded highly significant positive heterosis and other two crosses $\mathrm{P}_{1} \times \mathrm{P}_{4}$ and $\mathrm{P}_{2} \times \mathrm{P}_{1}$ showed nonsignificant positive relative heterosis. The minimum and maximum heterobeltiosis were observed in $\mathrm{P}_{3} \times \mathrm{P}_{4}$ (5.47 percent) and $\mathrm{P}_{3} \times \mathrm{P}_{1}$ (10.67 percent) and the hybrids $P_{3} \times P_{1}, P_{2} \times$ $\mathrm{P}_{3}, \mathrm{P}_{2} \times \mathrm{P}_{4}, \mathrm{P}_{4} \times \mathrm{P}_{3}, \mathrm{P}_{1} \times \mathrm{P}_{3}$ and $\mathrm{P}_{3} \times \mathrm{P}_{4}$ showed positive and significant heterobeltiosis. The heterosis percentage over standard variety varied from $\mathrm{P}_{3} \times \mathrm{P}_{2}$ (6.62) to $\mathrm{P}_{4} \times \mathrm{P}_{3}(14.87$ percent) and the hybrids namely $\mathrm{P}_{4} \times \mathrm{P}_{3}, \mathrm{P}_{1} \mathrm{x}$ $\mathrm{P}_{3}, \mathrm{P}_{3} \times \mathrm{P}_{4}, \mathrm{P}_{3} \times \mathrm{P}_{1}, \mathrm{P}_{2} \times \mathrm{P}_{3}, \mathrm{P}_{4} \times \mathrm{P}_{1}, \mathrm{P}_{4} \times \mathrm{P}_{2}$ and $\mathrm{P}_{2} \times \mathrm{P}_{4}$ recorded highly significant positive standard heterosis. Hybrids $\mathrm{P}_{3} \times \mathrm{P}_{2}$ showed significant and positive heterosis (Table 6).

Number of pods per plant varied from (23.79) $\mathrm{P}_{2}$ to (37.70) $\mathrm{P}_{4}$. The parents $\mathrm{P}_{4}, \mathrm{P}_{3}, \mathrm{P}_{1}$ and $\mathrm{P}_{2}$ recorded significantly more number of pods per plant than their grand mean (29.92). For this trait the minimum number of pods was recorded in the hybrid $\mathrm{P}_{1} \times \mathrm{P}_{4}$ (28.50) and maximum in the hybrid $\mathrm{P}_{4} \times \mathrm{P}_{2}$ (39.19) and out of 12 hybrids, nine hybrids viz., $\mathrm{P}_{4} \times \mathrm{P}_{2}$, $\mathrm{P}_{3} \times \mathrm{P}_{4}, \mathrm{P}_{4} \times \mathrm{P}_{3}, \mathrm{P}_{3} \times \mathrm{P}_{1}, \mathrm{P}_{4} \times \mathrm{P}_{1}, \mathrm{P}_{2} \times \mathrm{P}_{4}, \mathrm{P}_{1} \times$ $\mathrm{P}_{3}, \mathrm{P}_{1} \times \mathrm{P}_{2}$ and $\mathrm{P}_{1} \times \mathrm{P}_{4}$ exhibited significantly higher mean value when compared to their grand mean (34.93). For number of pods per plant, the $g c a$ values fell between $\mathrm{P}_{2}(-1.806)$ and $\mathrm{P}_{4}$ (2.661). The parents $\mathrm{P}_{4}$ and $\mathrm{P}_{3}$ recorded significant and positive effect and $\mathrm{P}_{1}$ and $\mathrm{P}_{2}$ registered negative significant for this trait. The $s c a$ effects varied from $\mathrm{P}_{1} \times \mathrm{P}_{4}$ (1.34) to $\mathrm{P}_{1} \times \mathrm{P}_{2}$ (3.18). With this trait the hybrids that showed significant and positive sca effects were $\mathrm{P}_{1} \times \mathrm{P}_{2}, \mathrm{P}_{4} \times \mathrm{P}_{2}, \mathrm{P}_{3} \times \mathrm{P}_{4}, \mathrm{P}_{2} \mathrm{x}$ $\mathrm{P}_{4}, \mathrm{P}_{1} \times \mathrm{P}_{3}$, and $\mathrm{P}_{2} \times \mathrm{P}_{3}$ and the hybrid $\mathrm{P}_{4} \times \mathrm{P}_{1}$, $\mathrm{P}_{3} \times \mathrm{P}_{2}, \mathrm{P}_{2} \times \mathrm{P}_{1}, \mathrm{P}_{3} \times \mathrm{P}_{1}$ and $\mathrm{P}_{1} \times \mathrm{P}_{4}$ registered negatively significant sca effects for number of pods per plant. 
Table.1 Mean performance of parents and hybrids

\begin{tabular}{|c|c|c|c|c|c|c|c|c|c|c|c|}
\hline Entries & $\begin{array}{c}\text { Days to } 50 \\
\text { per cent } \\
\text { flowering }\end{array}$ & $\begin{array}{l}\text { Days to } \\
\text { maturity }\end{array}$ & $\begin{array}{c}\text { Plant } \\
\text { height } \\
(\mathrm{cm})\end{array}$ & $\begin{array}{c}\text { No. of } \\
\text { branches } \\
\text { per } \\
\text { plant }\end{array}$ & $\begin{array}{c}\text { No. of } \\
\text { clusters } \\
\text { per } \\
\text { plant }\end{array}$ & $\begin{array}{c}\text { Pod } \\
\text { length } \\
(\mathrm{cm})\end{array}$ & $\begin{array}{c}\text { No. of } \\
\text { pods per } \\
\text { plant }\end{array}$ & $\begin{array}{c}\text { No. of } \\
\text { seeds } \\
\text { per } \\
\text { pod }\end{array}$ & $\begin{array}{l}100 \text { grain } \\
\text { weight } \\
\text { (g) }\end{array}$ & $\begin{array}{c}\text { Protein } \\
\text { content } \\
(\%)\end{array}$ & $\begin{array}{c}\text { Single } \\
\text { plant } \\
\text { yield }(g)\end{array}$ \\
\hline \multicolumn{12}{|c|}{ Parents } \\
\hline $\mathbf{P 1}$ & 35.66 & 65.66 & $28.15^{*}$ & 2.86 & 14.49 & 4.73 & $25.03 *$ & 6.05 & 4.78 & $20.05^{*}$ & 8.90 \\
\hline $\mathbf{P 2}$ & $34.33 *$ & $54.33 *$ & $25.97 *$ & $2.52 *$ & $12.39 *$ & $3.96 *$ & $23.79 *$ & 6.02 & 4.63 & $16.17 *$ & $7.13 *$ \\
\hline P3 & 36.00 & 66.00 & $37.15^{*}$ & 2.94 & 15.17 & 4.72 & $33.16^{*}$ & 6.14 & 4.95 & 17.70 & 8.67 \\
\hline $\mathbf{P 4}$ & 37.33 & 69.00 & $36.91 *$ & 3.21 & $16.98 *$ & 4.60 & $37.70 *$ & $6.84 *$ & $5.91 *$ & $19.35 *$ & $10.06 *$ \\
\hline \multicolumn{12}{|c|}{ Hybrids } \\
\hline P1 X P2 & 35.00 & 65.33 & $32.75 *$ & 3.67 & $23.50 *$ & $4.87 *$ & $29.90 *$ & 6.30 & 4.97 & $18.69 *$ & $8.29 *$ \\
\hline P1 X P3 & 35.66 & 65.00 & 39.90 & 3.69 & $19.00 *$ & 5.35 & $32.33 *$ & 6.19 & 5.95 & $17.14 *$ & $11.83 *$ \\
\hline P1X P4 & 37.00 & 68.00 & $33.42 *$ & 3.34 & $21.39 *$ & 4.94 & $28.50 *$ & 6.94 & 5.10 & 20.20 & 14.24 \\
\hline P2 X P1 & 35.00 & $62.33 *$ & $30.75 *$ & 3.35 & $17.39 *$ & $4.50 *$ & 35.16 & 6.44 & 5.83 & $18.63 *$ & $12.58 *$ \\
\hline P2 X P3 & $34.66 *$ & 65.00 & $33.15^{*}$ & $3.00 *$ & $22.09 *$ & 5.21 & 34.40 & 6.97 & 5.10 & $17.63 *$ & $11.01 *$ \\
\hline P2 X P4 & 35.66 & 66.00 & $41.56^{*}$ & 4.05 & $19.00 *$ & 5.05 & $33.55^{*}$ & 6.32 & 5.57 & 19.86 & $13.43 *$ \\
\hline P3 X P1 & 36.00 & 65.33 & $42.50 *$ & 3.83 & $16.5^{*}$ & $5.53 *$ & $36.87 *$ & $7.29 *$ & 5.27 & 20.48 & $14.98 *$ \\
\hline P3 X P2 & 35.00 & 66.66 & $43.29 *$ & 3.49 & $18.20 *$ & 5.04 & 35.18 & 6.87 & 5.49 & 20.65 & $13.19 *$ \\
\hline P3 X P4 & 36.00 & 65.00 & 39.26 & $4.19 *$ & $23.02 *$ & 5.27 & $39.13 *$ & 6.50 & $4.78^{*}$ & $19.06 *$ & $18.64 *$ \\
\hline P4 X P1 & 37.00 & 72.33 & $41.46^{*}$ & 3.97 & $17.59 *$ & 5.15 & $36.44 *$ & 6.52 & 5.61 & $17.54 *$ & $17.15^{*}$ \\
\hline P4 X P2 & 36.33 & 66.00 & $38.04 *$ & 3.57 & 19.39 & 5.12 & $39.19 *$ & 6.63 & $6.03 *$ & $21.15^{*}$ & 14.19 \\
\hline P4 X P3 & 35.66 & 66.66 & $45.00 *$ & 4.08 & $21.50 *$ & $5.43 *$ & $38.50 *$ & 6.43 & 5.93 & 20.14 & $20.69 *$ \\
\hline Mean of parents & 35.83 & 63.75 & 32.05 & 3.31 & 14.76 & 4.50 & 29.92 & 6.07 & 5.07 & 18.32 & 8.69 \\
\hline Mean of hybrids & 35.77 & 66.14 & 39.43 & 3.69 & 19.88 & 5.13 & 34.93 & 6.62 & 5.47 & 20.26 & 14.19 \\
\hline SEd & 0.54 & 0.60 & 0.38 & 0.24 & 0.38 & 0.12 & 0.67 & 0.33 & 0.26 & 0.33 & 0.37 \\
\hline $\mathrm{CD}(\mathrm{P}=05)$ & 1.104 & 1.24 & 0.782 & 0.50 & 0.79 & 0.25 & 1.37 & 0.67 & 0.53 & 0.67 & 0.76 \\
\hline
\end{tabular}

* Significant at $5 \%$ level 
Table.2 General combining ability effects of parents for different traits

\begin{tabular}{|c|c|c|c|c|c|c|c|c|c|c|c|c|}
\hline Parents & $\begin{array}{c}\text { Days to 50 } \\
\text { per cent } \\
\text { flowering }\end{array}$ & $\begin{array}{c}\text { Days to } \\
\text { maturity }\end{array}$ & $\begin{array}{c}\text { Plant } \\
\text { height }\end{array}$ & $\begin{array}{c}\text { No. of } \\
\text { branches } \\
\text { per plant }\end{array}$ & $\begin{array}{c}\text { No. of } \\
\text { clusters } \\
\text { per plant }\end{array}$ & $\begin{array}{c}\text { Pod } \\
\text { length }\end{array}$ & $\begin{array}{c}\text { No. of } \\
\text { pods per } \\
\text { plant }\end{array}$ & $\begin{array}{c}\text { No. of } \\
\text { seeds } \\
\text { per pod }\end{array}$ & $\begin{array}{c}\text { 100 } \\
\text { grain } \\
\text { weight }\end{array}$ & $\begin{array}{c}\text { Protein } \\
\text { content }\end{array}$ & $\begin{array}{c}\text { Single } \\
\text { plant } \\
\text { yield }\end{array}$ \\
\hline \multicolumn{7}{|c|}{} \\
\hline Vamban 4 & 0.104 & $0.667^{*}$ & $-2.193^{*}$ & -0.038 & $-0.556^{*}$ & 0.007 & $-2.520^{*}$ & -0.056 & -0.081 & 0.070 & $-0.702^{*}$ \\
\hline Vamban 2 & $-0.729 *$ & $-3.042^{*}$ & $-2.895^{*}$ & $-0.215^{*}$ & $-0.556^{*}$ & $-0.253^{*}$ & $-1.806^{*}$ & -0.081 & -0.087 & $-0.408^{*}$ & $-1.942^{*}$ \\
\hline LBG 17 & -0.146 & 0.167 & $2.846^{*}$ & 0.034 & $0.232^{*}$ & $0.192^{*}$ & $1.665^{*}$ & 0.039 & -0.068 & $-0.216^{*}$ & $0.647^{*}$ \\
\hline CO 5 & $0.771^{*}$ & $2.208^{*}$ & $2.242^{*}$ & $0.218^{*}$ & $0.880^{*}$ & 0.054 & $2.661^{*}$ & 0.099 & $0.236^{*}$ & $0.554^{*}$ & $1.997^{*}$ \\
\hline SE(gi) & $\mathbf{0 . 1 1 7}$ & $\mathbf{0 . 1 3 1}$ & $\mathbf{0 . 0 8 2}$ & $\mathbf{0 . 0 5 3}$ & $\mathbf{0 . 0 8 3}$ & $\mathbf{0 . 0 2 6}$ & $\mathbf{0 . 1 4 5}$ & $\mathbf{0 . 0 7 2}$ & $\mathbf{0 . 0 5 6}$ & $\mathbf{0 . 0 8 0}$ & $\mathbf{0 . 0 7 1}$ \\
\hline
\end{tabular}

*Significant at $5 \%$ level

Table.3 Specific combining ability effects of hybrids for different traits

\begin{tabular}{|c|c|c|c|c|c|c|c|c|c|c|c|}
\hline Hybrids & $\begin{array}{l}\text { Days to } \\
50 \text { per } \\
\text { cent } \\
\text { flowering }\end{array}$ & $\begin{array}{l}\text { Days to } \\
\text { maturity }\end{array}$ & $\begin{array}{c}\text { Plant } \\
\text { height }\end{array}$ & $\begin{array}{l}\text { No. of } \\
\text { branches } \\
\text { per plant }\end{array}$ & $\begin{array}{c}\text { No. of } \\
\text { clusters } \\
\text { per plant }\end{array}$ & $\begin{array}{c}\text { Pod } \\
\text { length }\end{array}$ & $\begin{array}{c}\text { No. of } \\
\text { pods per } \\
\text { plant }\end{array}$ & $\begin{array}{c}\text { No. of } \\
\text { seeds } \\
\text { per } \\
\text { pod }\end{array}$ & $\begin{array}{c}100 \\
\text { grain } \\
\text { weight }\end{array}$ & $\begin{array}{l}\text { Protein } \\
\text { content }\end{array}$ & $\begin{array}{c}\text { Single } \\
\text { plant } \\
\text { yield }\end{array}$ \\
\hline $\mathrm{P} 1 \times \mathrm{P} 2$ & -0.146 & $0.667 *$ & 0.007 & $0.278 *$ & $2.957 *$ & -0.038 & $3.180 *$ & -0.022 & $0.210^{*}$ & -0.031 & $0.266^{*}$ \\
\hline P1 X P3 & 0.104 & $-1.208^{*}$ & $3.717^{*}$ & $0.279 *$ & $-0.526^{*}$ & $0.275^{*}$ & $1.779 *$ & 0.227 & $0.392 *$ & -0.075 & $0.696^{*}$ \\
\hline P1 X P4 & $0.354 *$ & $1.750 *$ & $0.566^{*}$ & -0.011 & $0.569 *$ & 0.017 & $-1.349 *$ & 0.157 & -0.167 & $-0.779 *$ & $1.592 *$ \\
\hline P2 X P1 & 0.201 & $1.500 *$ & 0.998* & 0.157 & $3.052 *$ & $0.187^{*}$ & $-2.630 *$ & -0.068 & $-0.430 *$ & 0.032 & $-2.143^{*}$ \\
\hline P2 X P3 & -0.063 & $3.167 *$ & $1.441 *$ & -0.062 & $1.872 *$ & $-0.498 *$ & $1.255^{*}$ & $0.435^{*}$ & 0.078 & $0.738 *$ & $0.585^{*}$ \\
\hline P2 X P4 & 0.187 & $1.292 *$ & $3.621 *$ & $0.319 *$ & 0.270 & $0.317 *$ & $1.837 *$ & -0.068 & $0.284 *$ & $1.331 *$ & $0.947 *$ \\
\hline P3X P1 & -0.167 & -0.167 & $-1.298 *$ & -0.070 & $1.253 *$ & $-0.088 *$ & $-2.270 *$ & $-0.550 *$ & $0.340 *$ & $-1.670^{*}$ & $-1.573^{*}$ \\
\hline P3 X P2 & -0.175 & $-0.833^{*}$ & $-5.073 *$ & $-0.245^{*}$ & $1.945 *$ & $0.085 *$ & $-3.843^{*}$ & 0.050 & -0.197 & $-1.512 *$ & $-1.088^{*}$ \\
\hline P3 X P4 & $-0.562 *$ & $-2.083 *$ & $0.212 *$ & $0.400 *$ & $2.544 *$ & $0.137 *$ & $0.810^{*}$ & -0.201 & -0.182 & 0.230 & $4.209 *$ \\
\hline P4 X P1 & -0.015 & $-2.167 *$ & $-4.020 *$ & $-0.317^{*}$ & $1.900 *$ & $-0.108^{*}$ & $-3.968 *$ & 0.210 & $-0.285^{*}$ & $1.330 *$ & $-1.455^{*}$ \\
\hline P4 X P2 & $-0.333^{*}$ & -0.071 & $1.760 *$ & $0.240 *$ & -0.198 & -0.032 & $-2.822 *$ & -0.157 & $-0.223^{*}$ & $-0.648^{*}$ & $-0.380^{*}$ \\
\hline P4 X P3 & 0.167 & $-0.833^{*}$ & $-2.868 *$ & 0.057 & $0.760 *$ & $-0.080 *$ & 0.315 & 0.033 & $-0.577 *$ & $-0.540 *$ & $-1.028 *$ \\
\hline $\operatorname{SE}\left(S_{i j}\right)$ & 0.210 & 0.240 & 0.151 & 0.096 & 0.153 & 0.048 & 0.266 & 0.133 & 0.103 & 0.147 & 0.134 \\
\hline
\end{tabular}

* Significant at $5 \%$ level 
Table.4 Variability parameters for different traits

\begin{tabular}{|c|c|c|c|c|c|}
\hline S.No. & Characters & PCV (\%) & GCV (\%) & Heritability (\%) & Genetic advance as per cent of mean \\
\hline 1. & Days to 50 per cent flowering & 2.84 & 2.15 & 57.35 & 3.35 \\
\hline 2. & Days to maturity & 5.71 & 5.59 & 96.03 & 11.29 \\
\hline 3. & Plant height & 15.29 & 15.23 & 99.30 & 31.27 \\
\hline 4. & Number of branches per plant & 15.67 & 13.08 & 69.78 & 22.53 \\
\hline 5. & Number of clusters per plant & 17.11 & 16.92 & 97.78 & 34.46 \\
\hline 6. & Pod length & 8.41 & 7.83 & 86.83 & 15.04 \\
\hline 7. & Number of pods per plant & 14.23 & 14.02 & 97.04 & 28.46 \\
\hline 8. & Number of seeds per pod & 7.63 & 4.28 & 31.54 & 4.96 \\
\hline 9. & Hundred grain weight & 10.15 & 8.22 & 65.58 & 13.71 \\
\hline 10. & Protein content & 7.87 & 7.50 & 90.75 & 14.73 \\
\hline 11. & Single plant yield & 30.07 & 29.90 & 98.88 & 61.26 \\
\hline
\end{tabular}

Table.5 Percentage of heterosis for days to 50 percent flowering, Days to maturity, Plant height, Number of branches per plant

\begin{tabular}{|c|c|c|c|c|c|c|c|c|c|c|c|c|c|}
\hline \multirow[b]{2}{*}{$\begin{array}{c}\text { S.No } \\
\text {. }\end{array}$} & \multirow[b]{2}{*}{ Cross } & \multicolumn{3}{|c|}{ Days to 50 per cent flowering } & \multicolumn{3}{|c|}{ Days to maturity } & \multicolumn{3}{|c|}{ Plant height } & \multicolumn{3}{|c|}{ Number of branches per plant } \\
\hline & & $\begin{array}{l}\text { Relative } \\
\text { heterosis } \\
\text { (di) }\end{array}$ & \begin{tabular}{|c} 
Heterob \\
eltiosis \\
(dii)
\end{tabular} & $\begin{array}{c}\text { Standard } \\
\text { heterosis } \\
\text { (diii) }\end{array}$ & $\begin{array}{l}\text { Relative } \\
\text { heterosis } \\
\text { (di) }\end{array}$ & $\begin{array}{l}\text { Heterobelt } \\
\text { iosis (dii) }\end{array}$ & $\begin{array}{c}\text { Standard } \\
\text { heterosis } \\
\text { (diii) }\end{array}$ & $\begin{array}{l}\text { Relative } \\
\text { heterosis } \\
\text { (di) }\end{array}$ & $\begin{array}{c}\text { Heterobelt } \\
\text { iosis (dii) }\end{array}$ & $\begin{array}{c}\text { Standard } \\
\text { heterosis } \\
\text { (diii) }\end{array}$ & $\begin{array}{l}\text { Relative } \\
\text { heterosis } \\
\text { (di) }\end{array}$ & $\begin{array}{c}\text { Heterob } \\
\text { eltiosis } \\
\text { (dii) }\end{array}$ & $\begin{array}{c}\text { Standard } \\
\text { heterosis } \\
\text { (diii) }\end{array}$ \\
\hline 1 & P1 X P2 & -0.47 & -2.78 & $-6.25 * *$ & $8.59 * *$ & -1.01 & $-5.31 * *$ & $21.36 * *$ & $16.96 * *$ & $-11.85 * *$ & $32.89 * *$ & $22.33 * *$ & 14.09 \\
\hline 2 & P1 X P3 & -0.93 & -0.93 & $-4.46 * *$ & -1.52 & -1.52 & $-5.80 * *$ & $22.49 * *$ & $7.46^{* *}$ & $7.46^{* *}$ & $24.89 * *$ & $23.11 * *$ & 14.82 \\
\hline 3 & P1X P4 & 0.91 & -0.89 & -0.89 & 0.74 & -1.45 & -1.45 & $2.98 * *$ & $-9.45 * *$ & $-10.03 * *$ & 7.45 & 3.83 & 3.83 \\
\hline 4 & $\mathrm{P} 2 \mathrm{X}$ P1 & 0.48 & -1.87 & $-6.25 * *$ & $4.18 * *$ & $-5.08 * *$ & $-9.66 * *$ & $13.57 * *$ & $9.22 * *$ & $-17.23 * *$ & 14.43 & 11.89 & 4.35 \\
\hline 5 & P2 X P3 & -0.95 & $-3.70 *$ & $-7.14 * *$ & $8.33^{* *}$ & -1.52 & $-5.80 * *$ & $4.98 * *$ & $-10.78 * *$ & $-10.78 * *$ & 1.01 & 0.00 & -6.74 \\
\hline 6 & P2 X P4 & 0.00 & $-4.46 * *$ & $-4.46^{* *}$ & $7.32 * *$ & $-4.35 * *$ & $-4.35 * *$ & $32.11 * *$ & $12.58 * *$ & $11.86^{* *}$ & $30.29 * *$ & $25.91 * *$ & $25.91 * *$ \\
\hline 7 & P3 X P1 & 0.47 & 0.00 & $-3.57 *$ & -0.76 & -1.01 & $-5.31 * *$ & $30.45 * *$ & $14.86^{* *}$ & $14.39 * *$ & $30.68 * *$ & $27.78 * *$ & $19.17 * *$ \\
\hline 8 & P3 X P2 & -0.47 & -2.78 & $-6.25^{* *}$ & $10.80 * *$ & 1.01 & $-3.38 * *$ & $37.51 * *$ & $17.02 * *$ & $16.54 * *$ & $26.37 * *$ & 16.33 & 8.50 \\
\hline 9 & P3 X P4 & -1.82 & $-3.57 *$ & $-3.57 *$ & $-3.70 * *$ & $-5.80 * *$ & $-5.80 * *$ & $6.24 * *$ & $6.12 * *$ & $5.68 * *$ & $35.01 * *$ & $30.47 * *$ & $30.47 * *$ \\
\hline 10 & P4 X P1 & 1.83 & 0.00 & -0.89 & $7.43^{* *}$ & $4.83^{* *}$ & $4.83^{* *}$ & $27.28 * *$ & $12.07 * *$ & $11.61 * *$ & $35.45^{* *}$ & $32.44 * *$ & $23.52 * *$ \\
\hline 11 & P4 X P2 & 1.87 & -1.80 & -2.68 & $7.03 * *$ & $-4.35 * *$ & $-4.35 * *$ & $20.81 * *$ & $2.81 *$ & $2.39 *$ & $29.27 * *$ & $19.00 * *$ & 10.98 \\
\hline & SE & 0.46 & 0.54 & 0.54 & 0.52 & 0.60 & 0.60 & 0.33 & 0.38 & 0.38 & 0.21 & 0.24 & 0.24 \\
\hline
\end{tabular}

* Significant at $5 \%$ level, ** Significant at $1 \%$ level. 
Table.6 Percentage of heterosis for Number of cluster per plant, pod length, Pod per plant, Number of seeds per pod

\begin{tabular}{|c|c|c|c|c|c|c|c|c|c|c|c|c|c|}
\hline \multirow[b]{2}{*}{ S.No. } & \multirow[b]{2}{*}{ Cross } & \multicolumn{3}{|c|}{ Number of cluster per plant } & \multicolumn{3}{|c|}{ pod length } & \multicolumn{3}{|c|}{ Pod per plant } & \multicolumn{3}{|c|}{ Number of seeds per pod } \\
\hline & & $\begin{array}{c}\text { Relative } \\
\text { heterosis } \\
\text { (di) }\end{array}$ & $\begin{array}{c}\text { Heterob } \\
\text { eltiosis } \\
\text { (dii) } \\
\end{array}$ & $\begin{array}{c}\text { Standard } \\
\text { heterosis } \\
\text { (diii) }\end{array}$ & $\begin{array}{c}\text { Relative } \\
\text { heterosis } \\
\text { (di) }\end{array}$ & $\begin{array}{l}\text { Heterobelti } \\
\text { osis (dii) }\end{array}$ & $\begin{array}{c}\text { Standard } \\
\text { heterosis } \\
\text { (diii) }\end{array}$ & $\begin{array}{c}\text { Relative } \\
\text { heterosis } \\
\text { (di) }\end{array}$ & $\begin{array}{l}\text { Heterobelti } \\
\text { osis (dii) }\end{array}$ & $\begin{array}{c}\text { Standard } \\
\text { heterosis } \\
\text { (diii) } \\
\end{array}$ & $\begin{array}{c}\text { Relative } \\
\text { heterosis } \\
\text { (di) }\end{array}$ & $\begin{array}{l}\text { Heterobel } \\
\text { tiosis (dii) }\end{array}$ & $\begin{array}{c}\text { Standard } \\
\text { heterosis } \\
\text { (diii) }\end{array}$ \\
\hline 1 & $\mathrm{P} 1 \mathrm{X} \mathrm{P} 2$ & $78.08 * *$ & $67.86 * *$ & $38.40 * *$ & $8.70 * *$ & -2.53 & 3.03 & $22.56 * *$ & $19.61 * *$ & $-20.69 * *$ & 4.85 & 4.65 & -7.85 \\
\hline 2 & P1 X P3 & $30.29 * *$ & $25.24 * *$ & $11.94 * *$ & $10.28 * *$ & $7.18 * *$ & $13.25 * *$ & $11.17 * *$ & -2.51 & $-14.24 * *$ & 1.92 & 0.70 & -9.50 \\
\hline 3 & P1X P4 & $38.13 * *$ & $26.01 * *$ & $26.01 * *$ & 2.85 & -1.20 & 4.44 & $-9.09 * *$ & $-24.40 * *$ & $-24.40 * *$ & 8.10 & 1.46 & 1.46 \\
\hline 4 & P2 X P1 & $31.35 * *$ & $20.06 * *$ & 2.45 & 3.09 & -4.86 & -4.86 & $43.44 * *$ & $40.48 * *$ & $-6.74 * *$ & 6.83 & 6.33 & -5.85 \\
\hline 5 & P2 X P3 & $62.61 * *$ & $45.60 * *$ & $30.13 * *$ & $19.53 * *$ & $10.37 * *$ & $10.22 * *$ & $20.37 * *$ & 3.74 & $-8.74 * *$ & $14.82 * *$ & $13.45 * *$ & 1.95 \\
\hline 6 & $\mathrm{P} 2 \mathrm{X} \mathrm{P} 4$ & $31.12 * *$ & $11.90 * *$ & $11.90 * *$ & $17.51 * *$ & $9.77 * *$ & $6.91 * *$ & $8.75 * *$ & $-11.02 * *$ & $-11.02 * *$ & -1.51 & -7.55 & -7.75 \\
\hline 7 & P3 X P1 & $11.90 * *$ & $10.00 * *$ & -2.83 & $13.74 * *$ & $10.67 * *$ & $16.98 * *$ & $27.08 * *$ & $11.74 * *$ & -2.20 & $20.93 * *$ & $20.36^{* *}$ & 6.58 \\
\hline 8 & P3 X P2 & $32.93 * *$ & $21.38 * *$ & $7.22 * *$ & $12.49 * *$ & 0.87 & $6.62 *$ & $23.88 * *$ & $6.61 * *$ & $-6.69 * *$ & $14.33 * *$ & $14.11 *$ & 0.49 \\
\hline 9 & P3 X P4 & $43.96 * *$ & $35.57 * *$ & $35.57 * *$ & $9.78 * *$ & $5.47 * *$ & $11.49 * *$ & $10.69 * *$ & $3.78 *$ & $3.78^{*}$ & 1.25 & -4.97 & -4.97 \\
\hline 10 & $\mathrm{P} 4 \mathrm{X} \mathrm{P} 1$ & $11.76 * *$ & 3.51 & 3.63 & $6.00 * *$ & 3.13 & $9.02 * *$ & $15.63 * *$ & $-4.11 *$ & -3.35 & -0.13 & -6.87 & -4.68 \\
\hline 11 & P4 X P2 & $31.98 * *$ & $14.10 * *$ & $14.23 * *$ & $14.20 * *$ & 2.40 & $8.25 * *$ & $26.85 * *$ & 3.14 & $3.95^{*}$ & 1.92 & -5.19 & -2.97 \\
\hline 12 & P4 X P3 & $33.64 * *$ & $26.47 * *$ & $26.62 * *$ & $11.76^{* *}$ & $8.67 * *$ & $14.87 * *$ & $8.20 * *$ & 1.32 & 2.11 & -2.13 & -8.10 & -5.95 \\
\hline & SE & 0.33 & 0.38 & 0.38 & 0.10 & 0.12 & 0.12 & 0.58 & 0.67 & 0.67 & 0.29 & 0.33 & 0.33 \\
\hline
\end{tabular}

$*$ Significant at $5 \%$ level, $* *$ Significant at $1 \%$ level.

Table.7 Percentage of heterosis for Hundred grain weight, Protein content and Single plant yield

\begin{tabular}{|c|c|c|c|c|c|c|c|c|c|c|}
\hline \multirow[b]{2}{*}{ S.No. } & \multirow[b]{2}{*}{ Cross } & \multicolumn{3}{|c|}{ Hundred grain weight } & \multicolumn{3}{|c|}{ Protein content } & \multicolumn{3}{|c|}{ Single plant yield } \\
\hline & & $\begin{array}{c}\text { Relative } \\
\text { heterosis (di) }\end{array}$ & $\begin{array}{c}\text { Heterobelti } \\
\text { osis (dii) }\end{array}$ & $\begin{array}{c}\text { Standard } \\
\text { heterosis (diii) }\end{array}$ & $\begin{array}{c}\text { Relative } \\
\text { heterosis (di) }\end{array}$ & $\begin{array}{l}\text { Heterobeltio } \\
\text { sis (dii) }\end{array}$ & $\begin{array}{c}\text { Standard } \\
\text { heterosis (diii) }\end{array}$ & $\begin{array}{c}\text { Relative } \\
\text { heterosis (di) }\end{array}$ & $\begin{array}{c}\text { Heterobeltiosis } \\
\text { (dii) }\end{array}$ & $\begin{array}{c}\text { Standard } \\
\text { heterosis (diii) }\end{array}$ \\
\hline 1 & P1 X P2 & 3.29 & -0.47 & $-15.79 * *$ & 3.34 & $-6.53 * *$ & $-6.80^{* *}$ & 2.81 & $-7.85 *$ & $-17.56^{* *}$ \\
\hline 2 & P1 X P3 & $19.73 * *$ & $19.13 * *$ & 0.79 & $-9.09 * *$ & $-14.30 * *$ & $-14.54 * *$ & $33.95 * *$ & $31.48 * *$ & $17.63 * *$ \\
\hline 3 & P1X P4 & -6.39 & $-13.59 * *$ & $-13.59 * *$ & 2.63 & 1.03 & 0.75 & $49.49 * *$ & $41.62 * *$ & $41.62 * *$ \\
\hline 4 & P2 X P1 & $19.28 * *$ & $16.73 * *$ & -1.24 & 3.34 & $-7.11 * *$ & $-7.11 * *$ & $58.17 * *$ & $41.24 * *$ & $25.05^{* *}$ \\
\hline 5 & P2 X P3 & 2.51 & 2.00 & $-13.71 * *$ & 4.63 & -0.41 & $-12.08 * *$ & $40.61 * *$ & $27.07 * *$ & 9.51 \\
\hline 6 & $\mathrm{P} 2 \mathrm{X} \mathrm{P} 4$ & 2.23 & -5.64 & -5.64 & $12.34 * *$ & 2.60 & -0.98 & $57.52 * *$ & $33.57 * *$ & $33.57 * *$ \\
\hline 7 & P3 X P1 & 7.83 & 5.53 & $10.72 *$ & $7.63 * *$ & 2.11 & 2.11 & $67.31 * *$ & $66.44 * *$ & $48.91 * *$ \\
\hline 8 & P3 X P2 & $14.01^{* *}$ & 9.87 & -7.05 & $20.88^{* *}$ & $14.76^{* *}$ & 2.99 & $63.55^{* *}$ & $46.59 * *$ & $31.15^{* *}$ \\
\hline 9 & P3 X P4 & $-12.31 * *$ & $-19.06 * *$ & $-19.06 * *$ & 2.04 & -1.53 & $-4.97 *$ & $95.59 * *$ & $85.29 * *$ & $85.29 * *$ \\
\hline 10 & P4 X P1 & 4.14 & -6.39 & -4.96 & $-10.15 * *$ & $-12.51 * *$ & $-12.51 * *$ & $81.49 * *$ & $71.57 * *$ & $70.54 * *$ \\
\hline 11 & P4 X P2 & $13.51 * *$ & 0.61 & 2.14 & $20.29 * *$ & $11.35^{* *}$ & $5.48 * *$ & $65.72 * *$ & $41.97 * *$ & $41.12 * *$ \\
\hline \multirow[t]{2}{*}{12} & P4 X P3 & 8.43 & -1.06 & 0.45 & $9.73 * *$ & $6.00 * *$ & 0.42 & $121.71 * *$ & $106.97 * *$ & $105.73 * *$ \\
\hline & SE & 0.22 & 0.26 & 0.26 & 0.32 & 0.37 & 0.37 & 0.28 & 0.33 & 0.33 \\
\hline
\end{tabular}

* Significant at $5 \%$ level, ** Significant at $1 \%$ level. 
Table.8 Hybrids selected for recombination breeding

\begin{tabular}{|c|c|c|c|c|c|c|}
\hline $\begin{array}{l}\text { S.n } \\
\text { o }\end{array}$ & $\begin{array}{l}\text { Character } \\
\text { s }\end{array}$ & Mean & $\begin{array}{l}\text { Non- } \\
\text { significant } \\
\text { of } \\
\text { gca } \text { effects }\end{array}$ & $\begin{array}{l}\text { Significant of } \\
\text { sca effects }\end{array}$ & Standard heterosis & $\begin{array}{l}\text { Overall performance } \\
\text { of mean, } \\
\text { Non-significant of } \\
\text { gca effects, sca } \\
\text { effects and standard } \\
\text { heterosis }\end{array}$ \\
\hline 1. & $\begin{array}{l}\text { Days to } \\
50 \text { per } \\
\text { cent } \\
\text { flowering }\end{array}$ & VBN 2 x LBG 17 & $\begin{array}{l}\text { VBN } 4, \\
\text { LBG } 17, \text { CO } \\
5\end{array}$ & $\begin{array}{l}\text { VBN } 4 \text { x CO } 5, \text { LBG } 17 \text { x CO } 5, \\
\text { CO } 5 \text { x VBN } 2\end{array}$ & $\begin{array}{l}\text { VBN } 4 \times \text { VBN } 2, \text { VBN } 4 \times \text { LBG } 17, \\
\text { VB,N } 2 \times \text { VBN 4,VBN } 2 \times \text { LBG } 17, \\
\text { VBN } 2 \times \text { CO 5, LBG } 17 \times \text { VBN } 4 \text {, } \\
\text { LBG } 17 \times \text { VBN 2, LBG } 17 \times \text { CO } 5 \\
\text { CO } 5 \times \text { LBG } 17\end{array}$ & LBG 17x CO 5 \\
\hline 2. & $\begin{array}{l}\text { Days to } \\
\text { maturity }\end{array}$ & VBN 2 x VBN 4 & $\begin{array}{l}\text { VBN } 4, \\
\text { LBG } 17, \text { CO } \\
5\end{array}$ & 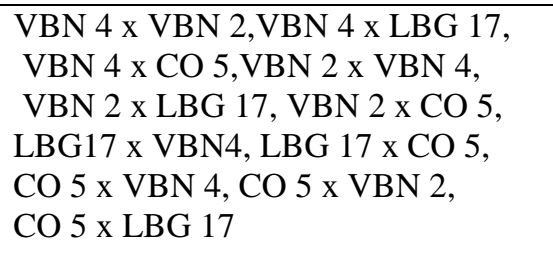 & $\begin{array}{l}\text { VBN } 4 \text { x VBN } 2, \text { VBN } 4 \text { x LBG } 17, \\
\text { VBN } 4 \text { x CO } 5, \text { VBN } 2 \text { x VBN } 4, \\
\text { VBN } 2 \times \text { LBG } 17, \text { VBN } 2 \times \text { CO } 5, \\
\text { LBG } 17 \times \text { VBN } 4, \text { LBG } 17 \times \text { VBN2, } \\
\text { LBG } 17 \times \text { CO } 5, \text { CO } 5 \times \text { VBN } 4, \\
\text { CO } 5 \times \text { VBN } 2, \text { CO } 5 \times \text { LBG } 17\end{array}$ & $\begin{array}{l}\text { VBN } 4 \text { x LBG } \\
17, \text { VBN } 4 \text { x CO 5, } \\
\text { LBG } 17 \times \text { VBN } 4, \\
\text { LBG } 17 \times \text { CO } 5, \\
\text { CO } 5 \times \text { VBN } 4, \text { CO } 5 \\
x \text { LBG } 17\end{array}$ \\
\hline 3. & $\begin{array}{l}\text { Plant } \\
\text { height }\end{array}$ & $\begin{array}{l}\text { VBN } 4 \text { x VBN2, VBN } 4 \text { x CO 5, } \\
\text { VBN } 2 \text { x VBN4, VBN } 2 \text { x LBG } 17, \\
\text { VBN } 2 \times \text { CO 5, LBG17x VBN4, LBG } \\
17 \times \text { VBN } 2, \text { CO } 5 \text { x VBN } 4, \text { CO } 5 \text { x } \\
\text { VBN } 2, \text { CO5 x LBG } 17\end{array}$ & - & $\begin{array}{l}\text { VBN } 4 \text { x VBN } 2, \text { VBN } 4 \text { x LBG } 17, \\
\text { VBN } 4 \text { x CO } 5, \text { VBN } 2 \text { x VBN } 4, \\
\text { VBN } 2 \text { x LBG } 17, \text { VBN } 2 x \text { CO } 5, \\
\text { LBG } 17 x \text { VBN } 4, \text { LBG } 17 x \text { VBN } 2, \\
\text { LBG } 17 x \text { CO } 5, \text { CO } 5 \text { x VBN } 4, \\
\text { CO } 5 \text { x VBN } 2, \text { CO } 5 \text { x LBG } 17\end{array}$ & 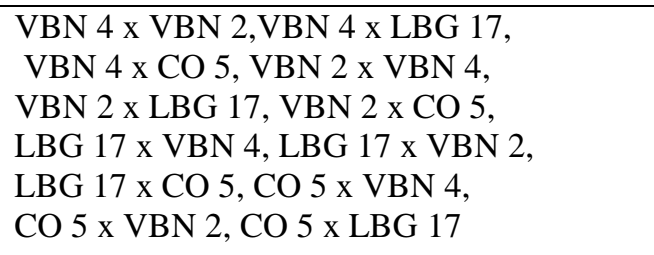 & - \\
\hline 4. & $\begin{array}{l}\text { Number } \\
\text { of } \\
\text { branches } \\
\text { per plant }\end{array}$ & $\begin{array}{l}\text { VBN } 2 \text { x LBG 17, } \\
\text { LBG } 17 \text { x CO5, }\end{array}$ & $\begin{array}{l}\text { VBN } 4, \\
\text { LBG } 17, \mathrm{CO} \\
5\end{array}$ & $\begin{array}{l}\text { VBN } 4 \text { x VBN 2,VBN } 4 \text { x LBG } 17, \\
\text { VBN } 2 \text { x CO 5,LBG } 17 \times \text { VBN } 2, \\
\text { LBG } 17 \text { x CO 5, CO } 5 \text { x VBN } 4 \text {, } \\
\text { CO } 5 \text { x VBN } 2 .\end{array}$ & $\begin{array}{l}\text { VBN } 2 \times \text { CO } 5 \text {, LBG } 17 \times \text { VBN } 4 \text {, } \\
\text { LBG } 17 \times \text { CO } 5 \text {, CO } 5 \times \text { VBN } 4 \text {, } \\
\text { CO } 5 \times \text { LBG } 17\end{array}$ & $\begin{array}{l}\text { LBG } 17 \text { x CO 5, CO } 5 \\
\text { x VBN } 4\end{array}$ \\
\hline 5. & $\begin{array}{l}\text { Number } \\
\text { of clusters } \\
\text { per plant }\end{array}$ & $\begin{array}{l}\text { VBN } 4 \text { x VBN } 2, \text { VBN } 4 \times \text { LBG } 17, \\
\text { VBN } 4 \times \text { CO } 5 \text {, VBN } 2 \times \text { VBN } 4, \\
\text { VBN } 2 \times \text { LBG } 17, \text { VBN } 2 \times \text { CO } 5 \text {, } \\
\text { LBG } 17 \times \text { VBN } 4, \text { LBG } 17 \times \text { VBN } 2 \text {, } \\
\text { LBG } 17 \times \text { CO } 5, \text { CO } 5 \times \text { VBN } 4 \text {, } \\
\text { CO } 5 \times \text { LBG } 17 .\end{array}$ & $\begin{array}{l}\text { VBN 4,LBG } \\
17\end{array}$ & $\begin{array}{l}\text { VBN } 4 \text { x VBN } 2, \text { VBN } 4 \text { x LBG } 17, \\
\text { VBN } 4 \text { x CO 5, VBN } 2 \times \text { VBN } 4, \\
\text { VBN } 2 \text { x LBG 17, LBG } 17 \text { x VBN } 4 \text {, } \\
\text { LBG } 17 \text { x VBN 2, LBG } 17 \times \text { CO 5, } \\
\text { CO } 5 \text { x LBG } 17 .\end{array}$ & $\begin{array}{l}\text { VBN } 4 \text { x VBN2,VBN } 4 \text { x LBG } 17, \text { VBN } 4 \text { x } \\
\text { CO 5,VBN } 2 \text { x LBG } 17 \text {, VBN } 2 \text { x CO5,LBG } \\
17 \text { x VBN } 2 \text {, LBG } 17 \text { x CO } 5 \text {, CO } 5 \text { x VBN } \\
2, \\
\text { CO } 5 \text { x LBG } 17 .\end{array}$ & VBN 4 x LBG 17 \\
\hline
\end{tabular}


Table.9 Hybrids selected for recombination breeding

\begin{tabular}{|c|c|c|c|c|c|c|}
\hline S.no & Characters & Mean & $\begin{array}{l}\text { Non- } \\
\text { significant of } \\
\text { gca effects }\end{array}$ & $\begin{array}{l}\text { Significant of } \\
\text { sca effects }\end{array}$ & Standard heterosis & $\begin{array}{l}\text { Overall } \\
\text { performance } \\
\text { of mean, } \\
\text { Non- } \\
\text { significant of } \\
\text { gca effects, sca } \\
\text { effects and } \\
\text { standard } \\
\text { heterosis }\end{array}$ \\
\hline 6. & Pod length & $\begin{array}{l}\text { VBN } 4 \text { x VBN } 2 \text {, VBN } 2 \times \text { VBN } \\
4, \text { LBG } 17 x \text { VBN } 4, \text { CO5 x LBG } \\
17 .\end{array}$ & $\begin{array}{l}\text { VBN } 4, \\
\text { LBG } 17, \text { CO } 5\end{array}$ & $\begin{array}{l}\text { VBN } 4 \text { x LBG } 17, \text { VBN } 2 \text { x } \\
\text { VBN } 4, \\
\text { VBN } 2 \text { x LBG 17, VBN } 2 \text { x CO } \\
5 .\end{array}$ & $\begin{array}{l}\text { VBN } 4 \text { x LBG } 17, \text { VBN } 2 \text { xLBG } 17, \\
\text { VBN } 2 \times \text { CO } 5 \text {, LBG } 17 \times \text { VBN } 4, \\
\text { LBG } 17 \times \text { VBN } 2, \text { LBG } 17 \times \text { CO } 5 \text {, } \\
\text { CO } 5 \times \text { VBN } 4 \text {, CO } 5 \times \text { VBN } 2, \\
\text { CO } 5 \times \text { LBG } 17\end{array}$ & $\begin{array}{l}\text { VBN } 4 \text { x LBG } \\
17\end{array}$ \\
\hline 7. & $\begin{array}{l}\text { Number of pods per } \\
\text { plant }\end{array}$ & $\begin{array}{l}\text { VBN } 4 \text { x VBN2,VBN } 4 \text { x LBG } \\
17, \text { VBN } 4 \text { x CO } 5, \text { VBN } 2 \text { x } \\
\text { CO5 } \\
\text { LBG } 17 \times \text { VBN } 4, \text { LBG } 17 \times \text { CO } \\
5, \text { CO } 5 \text { x VBN } 4, \text { CO } 5 \times \text { VBN } \\
2, \\
\text { CO } 5 \text { x LBG } 17\end{array}$ & - & $\begin{array}{l}\text { VBN } 4 \text { x VBN } 2, \mathrm{VBN} 4 \text { x LBG } \\
17, \\
\text { VBN } 4 \text { x CO 5, VBN } 2 \text { x VBN } 4 \text {, } \\
\text { VBN 2x LBG17, VBN 2x CO 5, } \\
\text { LBG } 17 x \text { VBN 4, LBG } 17 x \text { VBN } \\
2, \\
\text { LBG } 17 x \text { CO 5, CO } 5 \text { x VBN } 4, \\
\text { CO } 5 \text { x VBN } 2\end{array}$ & $\begin{array}{l}\text { VBN } 4 \text { x VBN } 2, \text { VBN } 4 \text { x LBG } 17, \\
\text { VBN } 4 \text { x CO 5, VBN } 2 \times \text { VBN } 4, \\
\text { VBN } 2 \times \text { LBG } 17, \text { VBN } 2 \times \text { CO } 5, \\
\text { LBG } 17 \times \text { VBN } 2, \text { LBG } 17 \text { x CO } 5 \text {, } \\
\text { CO } 5 \times \text { VBN } 2\end{array}$ & - \\
\hline 8. & $\begin{array}{l}\text { Number of seeds per } \\
\text { pod }\end{array}$ & LBG 17x VBN 4 & $\begin{array}{l}\text { VBN 4,VBN 2, } \\
\text { LBG17 }\end{array}$ & $\begin{array}{l}\text { VBN } 2 \text { x LBG 17, LBG } 17 \text { x } \\
\text { VBN } 4\end{array}$ & - & $\begin{array}{l}\text { VBN 2x LBG } \\
17, \text { LBG 17x } \\
\text { VBN } 4\end{array}$ \\
\hline 9. & $\begin{array}{l}\text { Hundred grain } \\
\text { weight }\end{array}$ & LBG 17x CO 5, CO 5 x VBN 2 & $\begin{array}{l}\text { VBN 4,VBN 2, } \\
\text { LBG17 }\end{array}$ & $\begin{array}{l}\text { VBN } 4 \text { x VBN 2,VBN4 x LBG17, } \\
\text { VBN } 2 \text { x VBN 4,VBN } 2 \times \text { CO5, } \\
\text { CO5 x VBN } 4 \text {, CO5 x VBN2, } \\
\text { CO5 x LBG } 17\end{array}$ & $\begin{array}{l}\text { VBN } 4 \text { x VBN } 2 \text {, VBN } 4 \text { x CO } 5 \text {, } \\
\text { VBN2x LBG } 17, \text { LBG } 17 x \text { VBN } 4 \text {, } \\
\text { LBG } 17 x \text { CO } 5\end{array}$ & $\begin{array}{l}\text { VBN } 4 \times \text { VBN } \\
2\end{array}$ \\
\hline
\end{tabular}


Table.10 Hybrids selected for recombination breeding

\begin{tabular}{|c|c|c|c|c|c|c|}
\hline S.no & Characters & Mean & $\begin{array}{l}\text { Non- } \\
\text { significant of } \\
\text { gca } \text { effects }\end{array}$ & $\begin{array}{l}\text { Significant of } \\
\text { sca effects }\end{array}$ & Standard heterosis & $\begin{array}{l}\text { Overall performance of } \\
\text { mean, } \\
\text { Non-significant of } g c a \\
\text { effects, } s c a \text { effects and } \\
\text { standard heterosis }\end{array}$ \\
\hline 10. & $\begin{array}{l}\text { Protein } \\
\text { content }\end{array}$ & $\begin{array}{l}\text { VBN } 4 \text { XVBN } 2, \text { VBN } 4 \text { x LBG17, } \\
\text { VBN } 2 \text { x VBN } 4, \text { VBN } 2 \text { x LBG } 17 \\
\text { LBG } 17 x \text { CO } 5, \text { CO } 5 \text { x VBN } 4, \\
\text { CO } 5 \text { x VBN } 2\end{array}$ & LBG17 & $\begin{array}{l}\text { VBN } 4 \text { x CO 5, VBN 2x LBG 17, } \\
\text { VBN } 2 \text { x CO 5, LBG } 17 \text { x VBN 4, } \\
\text { LBG } 17 \text { x VBN } 2, \text { CO } 5 \text { XVBN 4, CO } \\
5 \text { XVBN } 2, \text { CO } 5 \text { x LBG } 17\end{array}$ & $\begin{array}{l}\text { VBN } 4 \text { XVBN } 2, \text { VBN } 4 \text { x } \\
\text { LBG17, VBN } 2 \text { x VBN } 4, \\
\text { VBN } 2 \text { x LBG 17, LBG } 17 x \\
\text { CO } 5, \text { CO } 5 \text { x VBN } 4, \\
\text { CO } 5 \text { x VBN } 2\end{array}$ & VBN 2 x LBG 17 \\
\hline 11. & $\begin{array}{l}\text { Single plant } \\
\text { yield }\end{array}$ & $\begin{array}{l}\text { VBN 4x VBN 2, VBN } 4 \text { x LBG17, } \\
\text { VBN } 2 \text { x VBN 4, VBN 2x LBG17, } \\
\text { VBN 2x CO 5, LBG 17x VBN 4, } \\
\text { LBG } 17 x \text { VBN 2, LBG 17x CO 5, } \\
\text { CO } 5 \text { x VBN 4, CO } 5 \text { x LBG } 17\end{array}$ & $\begin{array}{l}\text { VBN 4,LBG } \\
17\end{array}$ & $\begin{array}{l}\text { VBN } 4 \text { x VBN 2,VBN } 4 \text { x LBG } 17 \\
\text { VBN } 4 \text { x CO 5, VBN } 2 \text { x VBN } 4, \\
\text { VBN } 2 \text { x LBG 17, VBN } 2 \text { x CO 5, } \\
\text { LBG } 17 \text { x VBN } 4, \text { LBG } 17 \times \text { VBN } 2 \\
\text { LBG } 17 \times \text { CO 5, CO } 5 \text { x VBN } 4, \\
\text { CO } 5 \text { x VBN } 2, \text { CO5 x LBG } 17\end{array}$ & $\begin{array}{l}\text { VBN } 4 \text { x VBN 2,VBN } 4 \text { x } \\
\text { LBG } 17, \text { VBN } 4 \text { x CO } 5, \\
\text { VBN } 2 \text { x VBN 4, VBN } 2 \text { x } \\
\text { CO } 5, \text { LBG } 17 x \text { VBN } 4, \\
\text { LBG } 17 \text { x VBN 2, LBG } 17 \text { x } \\
\text { CO } 5, \\
\text { CO } 5 \text { x VBN4, CO } 5 \text { x VBN } \\
2, \\
\text { CO } 5 \text { x LBG } 17\end{array}$ & $\begin{array}{l}\text { VBN } 4 \text { x LBG 17, LBG 17x } \\
\text { VBN } 4\end{array}$ \\
\hline \multicolumn{2}{|c|}{ Over all effcet } & $\begin{array}{l}\text { VBN 4x VBN 2, VBN } 2 \text { x VBN } 4 \\
\text { VBN 2x LBG17, LBG } 17 x \text { VBN } 4, \\
\text { LBG } 17 x \text { CO } 5\end{array}$ & $\begin{array}{l}\text { LBG 17,VBN } \\
4\end{array}$ & $\begin{array}{l}\text { VBN } 2 \text { x LBG } 17, \text { VBN } 4 \text { x LBG } 17, \\
\text { VBN } 2 \text { x CO } 5, \text { LBG } 17 x \text { VBN } 4, \\
\text { LBG } 17 \times \text { CO } 5\end{array}$ & $\begin{array}{l}\text { LBG } 17 \text { x CO 5, VBN } 4 x \\
\text { VBN } 2 \\
\text { VBN } 4 \text { x LBG 17,VBN } 2 \times x \\
\text { LBG } 17, \text { VBN } 2 \text { x CO } 5,\end{array}$ & VBN 4 x LBG 17 \\
\hline
\end{tabular}


Table.11 Estimation of genetic parameters

\begin{tabular}{|l|c|c|c|c|c|c|}
\hline \multicolumn{1}{|c|}{ Characters } & $\mathbf{D}$ & $\mathbf{F}$ & $\mathbf{H}_{\mathbf{1}}$ & $\mathbf{H}_{\mathbf{2}}$ & $\mathbf{h}^{\mathbf{2}}$ & $\mathbf{E}$ \\
\hline Days to 50 per cent flowering & 1.00 & -0.42 & -0.7 & -0.56 & -0.36 & 0.51 \\
\hline Days to maturity & 40.97 & 26.78 & 27.12 & 22.19 & 12.32 & 0.68 \\
\hline Plant height (cm) & 33.31 & -1.49 & 49.05 & 49.09 & 90.99 & 0.62 \\
\hline No. of branches per plant & 0.02 & -0.09 & 0.628 & 0.65 & 1.40 & 0.05 \\
\hline No. of clusters per plant & 3.42 & 2.33 & 33.87 & 33.12 & 58.96 & 0.16 \\
\hline Pod length (cm) & 0.11 & -0.00 & 0.44 & 0.43 & 0.84 & 0.02 \\
\hline No. of pods per plant & 43.49 & 21.67 & 39.16 & 35.37 & 55.90 & 0.68 \\
\hline No. of seeds per pod & 0.07 & 0.10 & 0.23 & 0.21 & 0.22 & 0.07 \\
\hline 100 grain weight (g) & 0.29 & 0.26 & 0.41 & 0.35 & 0.34 & 0.03 \\
\hline Protein content (\%) & 2.63 & 3.84 & 4.59 & 3.33 & 1.71 & 0.38 \\
\hline Single plant yield (g) & 1.31 & -4.69 & 48.08 & 42.60 & 67.88 & 0.13 \\
\hline
\end{tabular}

* Significant at 5\% level, ** Significant at 1\% level, D - Additive effects of genes, F - Covariance of additive and dominance effects, $\mathrm{H}_{1}$ - Dominance effects of genes, $\mathrm{H}_{2}$ - Dominance indicating symmetry of positive and negative effects of genes, $\mathrm{h}^{2}$ - Dominance effects over all loci, E - Environmental component

Table.12 Qualitative traits

\begin{tabular}{|c|l|l|}
\hline Scales & Percentage of plant foliage affected & Reaction \\
\hline 1 & Mottling of leaves covering 0.1 to 5.0 per cent of the leaf area. & Resistant \\
\hline 3 & Mottling of leaves covering 5.1 to 10.0 per cent of the leaf area. & Moderately resistant \\
\hline 5 & Mottling and yellow discoloration of 10.1 to 25.0 per cent of the leaf area. & Moderately susceptible \\
\hline 7 & Mottling and yellow discoloration of 25.1to 50.0 per cent of the leaf area. & Susceptible \\
\hline 9 & Severe yellow mottling on more than 50.0 per cent and up to 100 per cent of the leaf area. & Highly susceptible \\
\hline
\end{tabular}

The mean disease scale of parents and $F_{1}$ was calculated as follows (Singh, 1980). Mean scale $=\sum$ (Infection rate $\mathrm{x}$ Frequency) / Total number of plants scored. The plants in the $F_{2}$ and back cross generations were classified as resistant (1-3) and susceptible (5-9) following Reddy and Singh (1993).

Table.13 YMV scores in parents and hybrids

\begin{tabular}{|l|l|l|l|}
\hline \multicolumn{1}{|c|}{ Genotypes } & Mean YMV score & \multicolumn{1}{c|}{ Reaction } \\
\hline \multicolumn{2}{|c|}{ Code no. } & \multicolumn{1}{l|}{} \\
\hline P1 & Vamban 4 & 1.0 & Resistant \\
\hline P2 & Vamban 2 & 1.0 & Resistant \\
\hline P3 & LBG 17 & 3.8 & Moderately resistant \\
\hline P4 & CO 5 & 9.0 & Highly Susceptible \\
\hline Hybrids & VBN4 X VBN2 & 1.2 & Resistant \\
\hline P1 X P2 & V 2 & Moderately resistant \\
\hline P1 X P3 & VBN4 X LBG 17 & 4.3 & Moderately resistant \\
\hline P1X P4 & VBN4 X CO 5 & 3.8 & Resistant \\
\hline P2 X P1 & VBN2 X VBN 4 & 1.8 & Moderately resistant \\
\hline P2 X P3 & VBN2 X LBG 17 & 3.4 & Susceptible \\
\hline P2 X P4 & VBN2 X CO 5 & 7.6 & Moderately resistant \\
\hline P3 X P1 & LBG 17 X VBN 4 & 4.2 & Resistant \\
\hline P3 X P2 & LBG 17 X VBN 2 & 1.5 & Moderately susceptible \\
\hline P3 X P4 & LBG 17 X CO5 & 5.8 & Moderately resistant \\
\hline P4 X P1 & CO 5 X VBN4 & 4.2 & Moderately resistant \\
\hline P4 X P2 & CO 5 X VBN 2 & 4.5 & Highly Susceptible \\
\hline P4 X P3 & C0 5 X LBG 17 & 9.2 & \\
\hline
\end{tabular}


Fig.1 Phenotypic Coefficient of Variation (PCV) and Genotypic Coefficient of Variation (GCV) for single plant yield \& its components

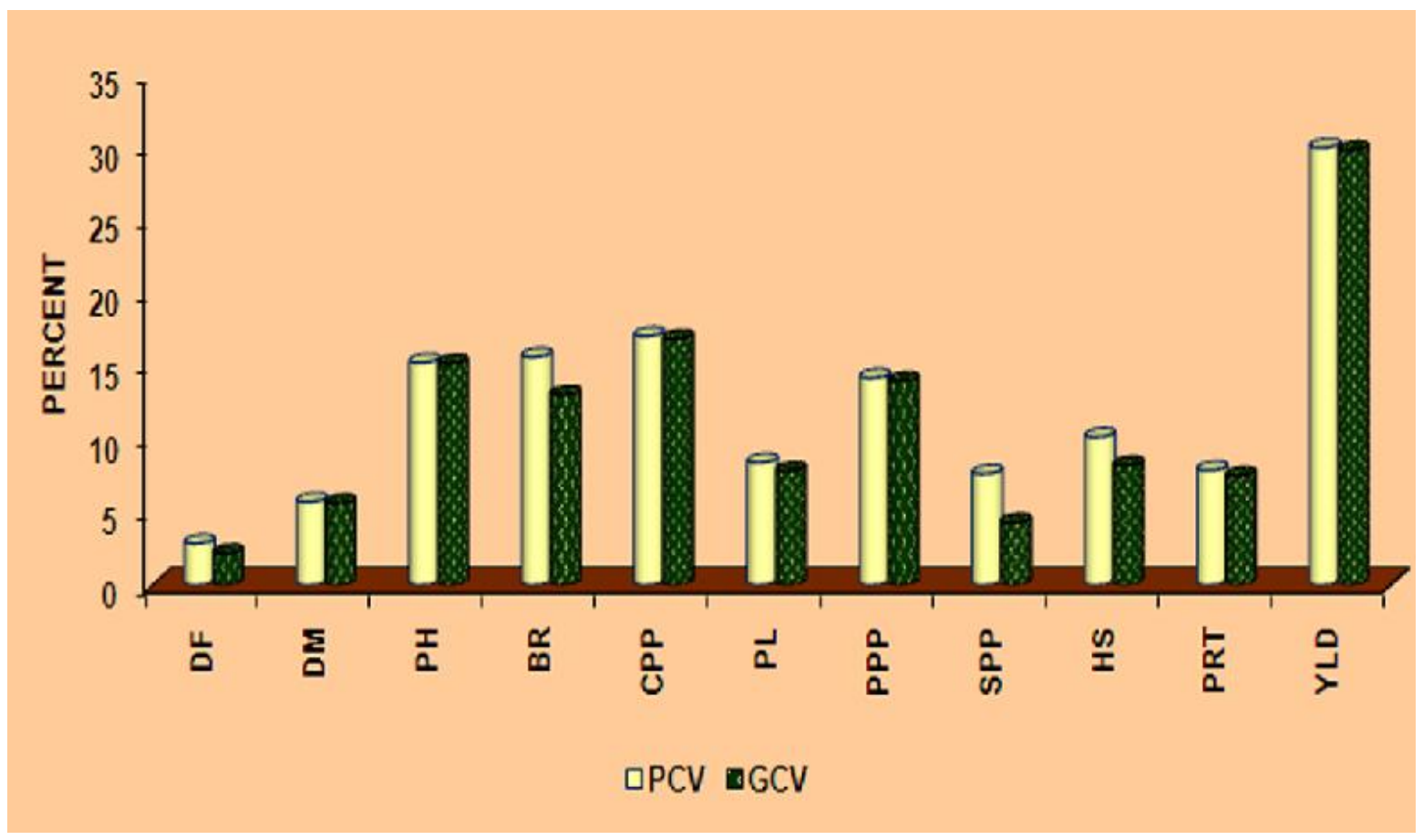

Fig.2 Heritability and Genetic Advance for single plant yield \& its components

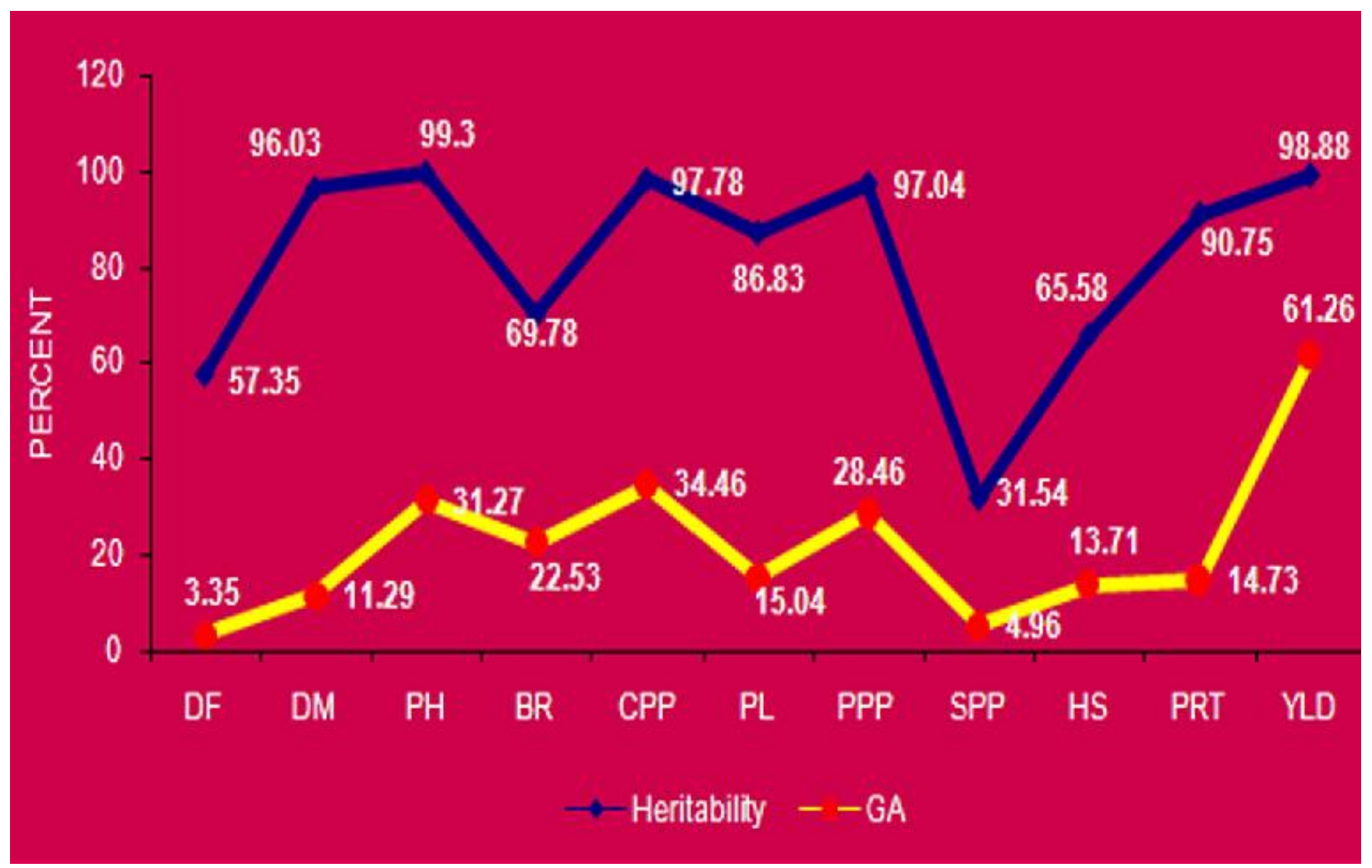


Fig.3 Range of sca effects of hybrids for different traits

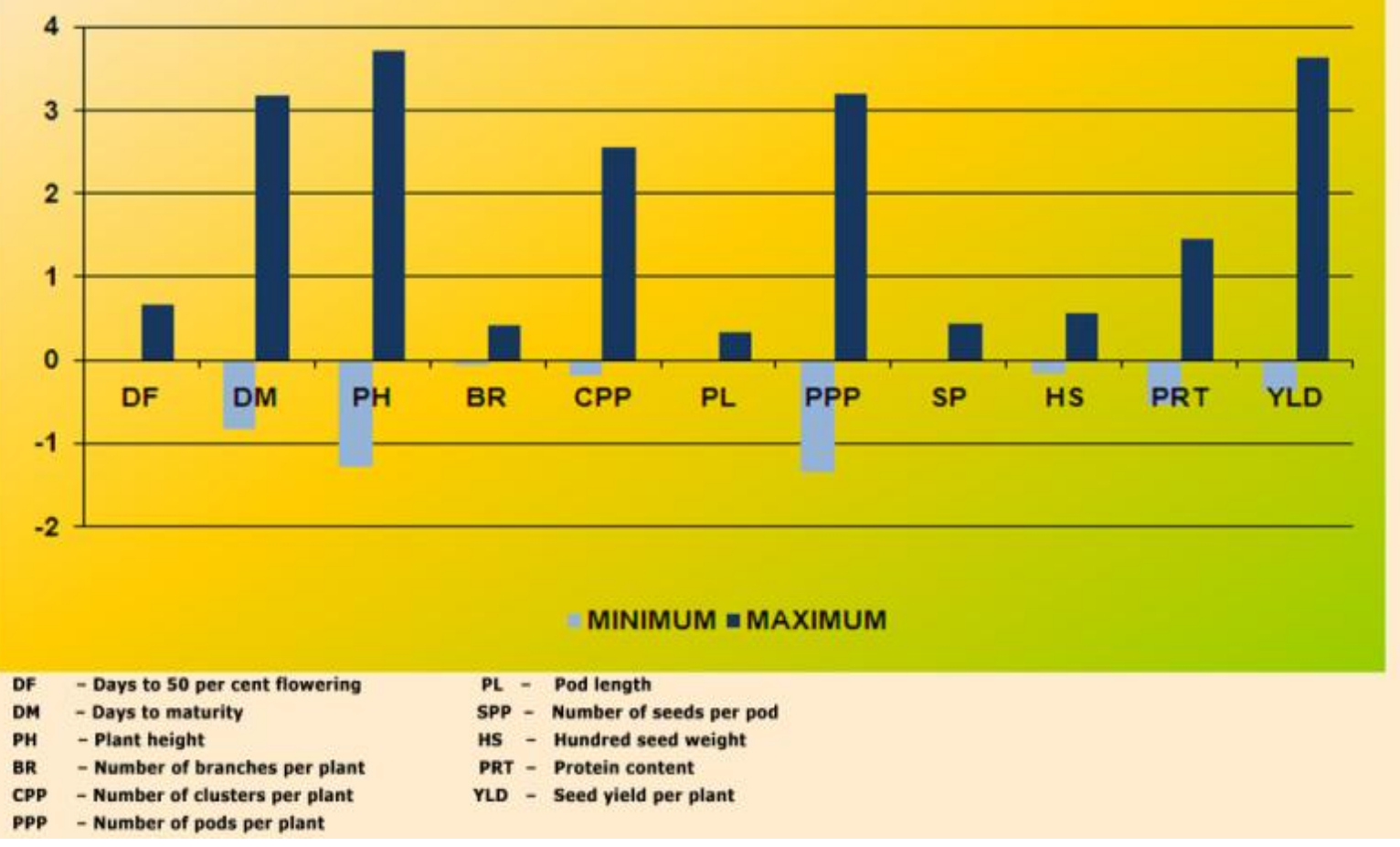

Fig.4 Range of relative heterosis for different traits

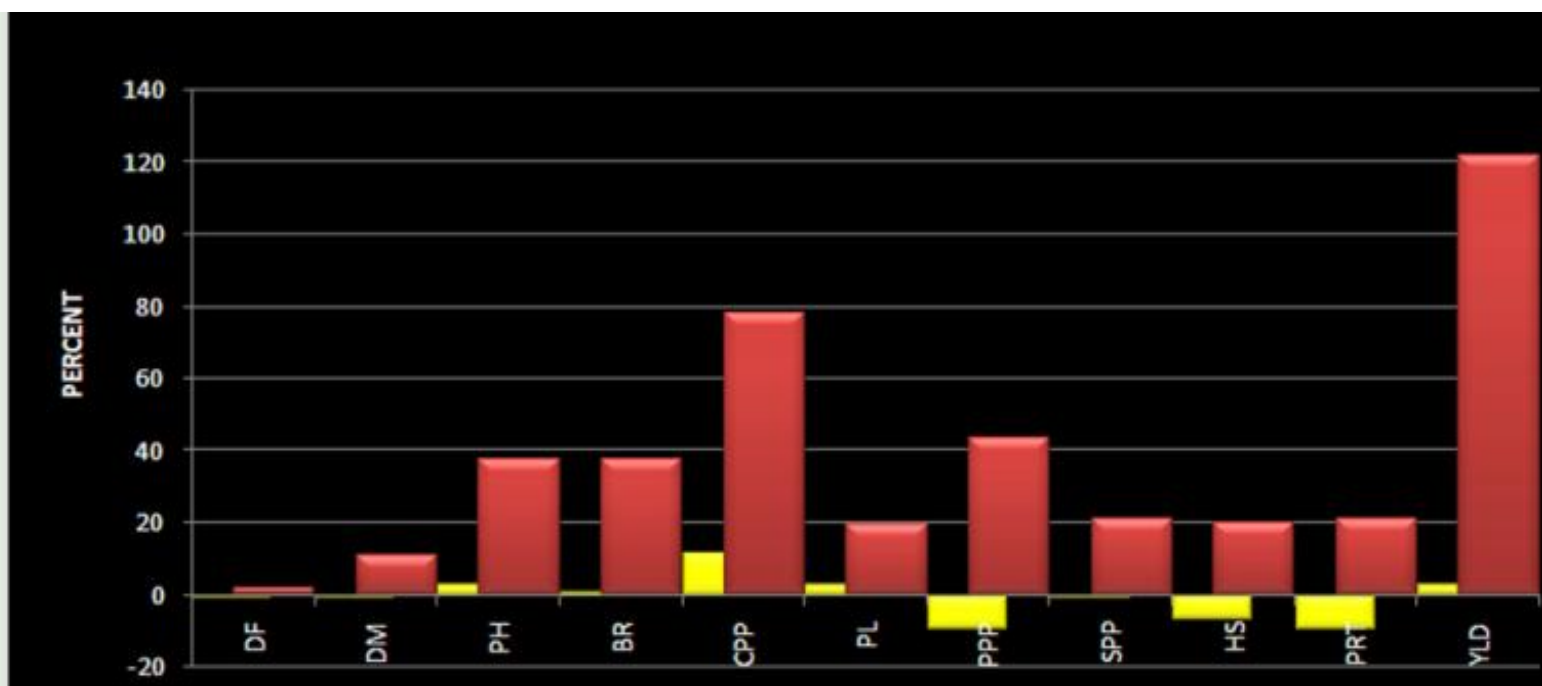

\section{- MINIMUM N MAXIMUM}

$\begin{array}{lll}\text { DF } & \text { - Days to } 50 \text { per cent flowering } & \text { PL - Pod length } \\ \text { DM } & \text { - Days to maturity } & \text { SPP - Number of seeds per pod } \\ \text { PH } & \text { - Plant height } & \text { HS - Hundred seed weight } \\ \text { BR } & \text { - Number of branches per plant } & \text { PRT - Protein content } \\ \text { CPP } & \text { - Number of clusters per plant } & \text { VLD - Seed yield per plant } \\ \text { PPP } & \text { - Number of pods per plant } & \end{array}$


Fig.5 Range of heterobeltiosis for different traits

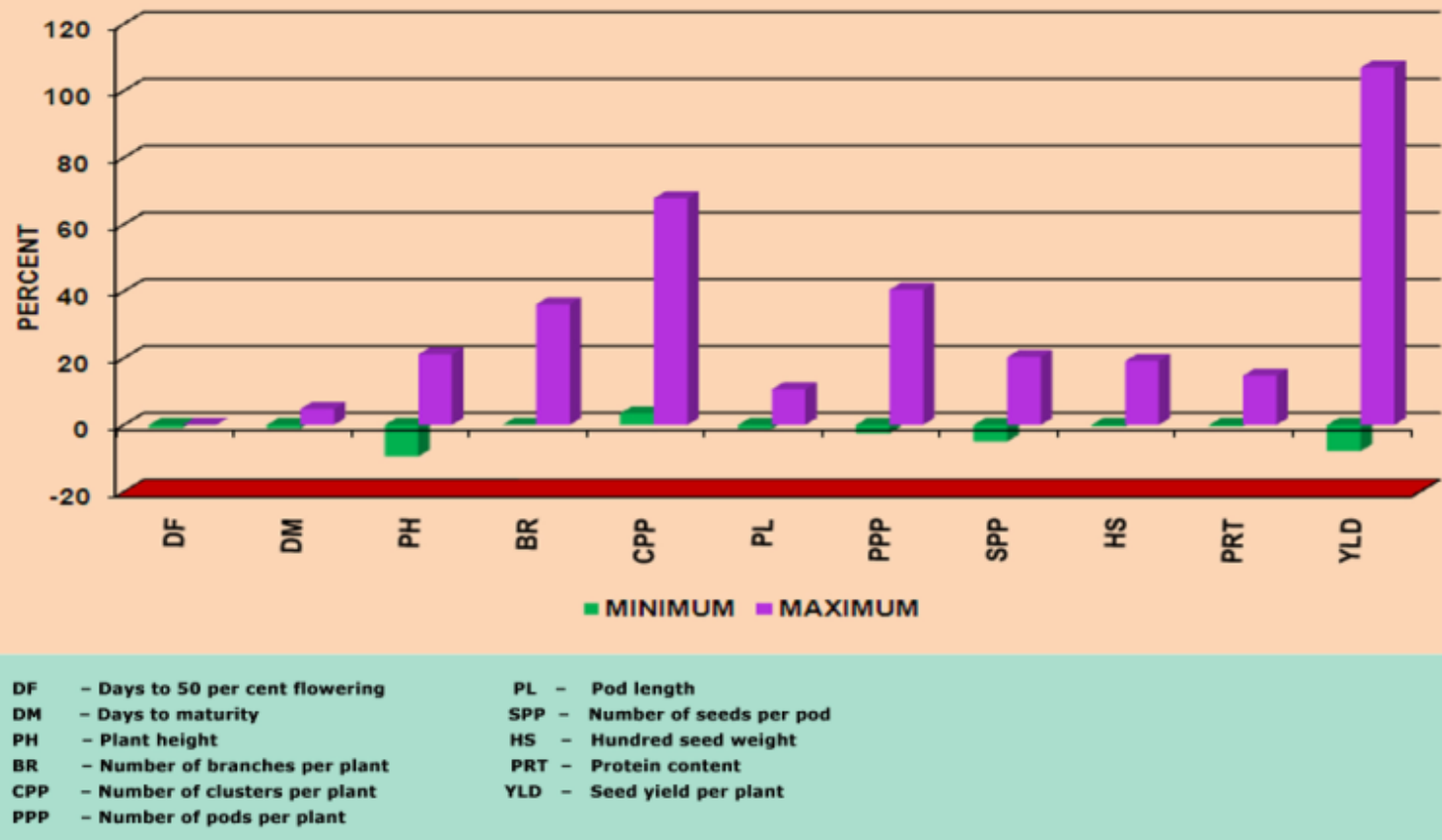

Fig.6 Range of standard heterosis for different traits

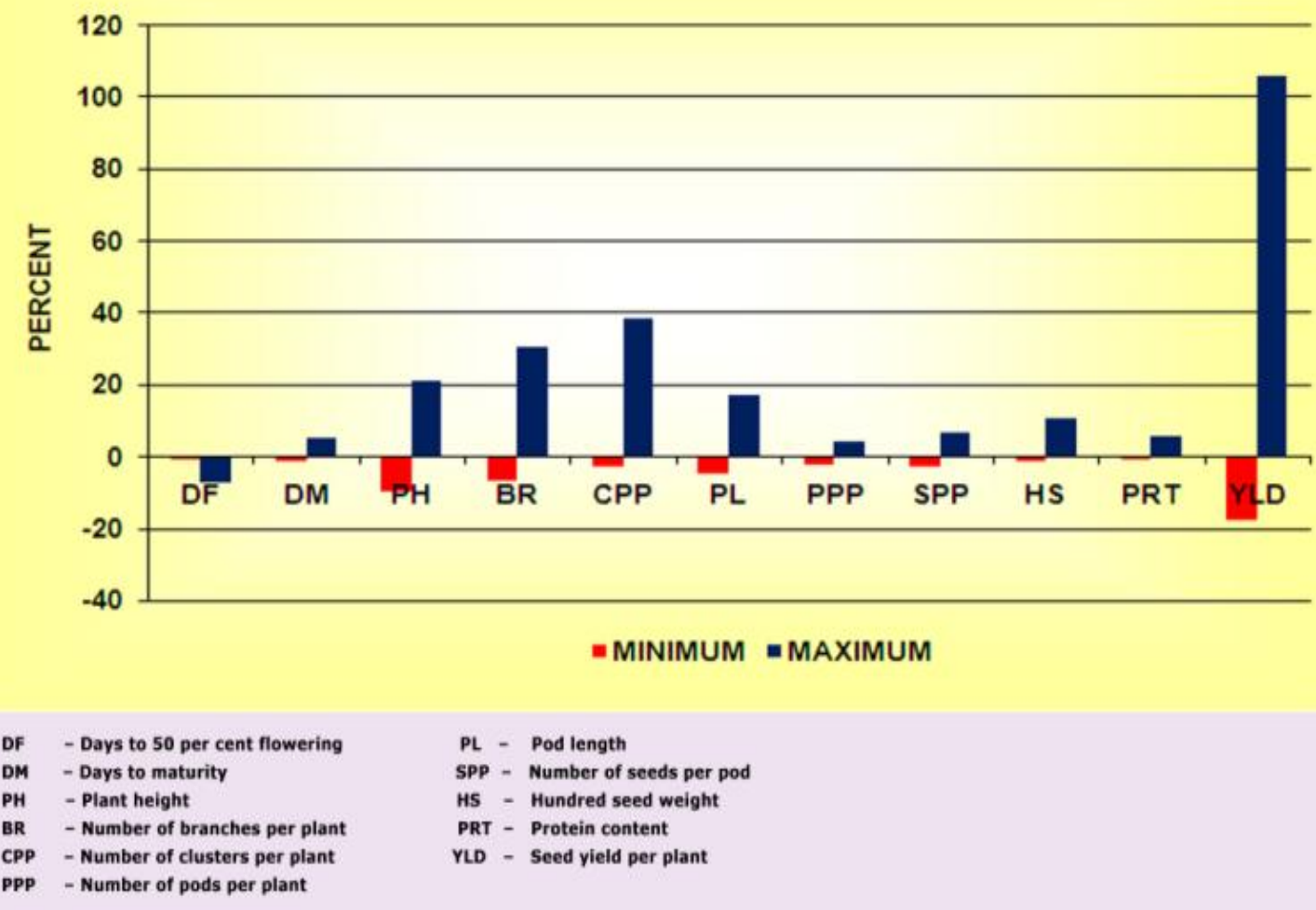


Fig.7 Magnitude of additive and dominance variance for single plant yield and its components traits

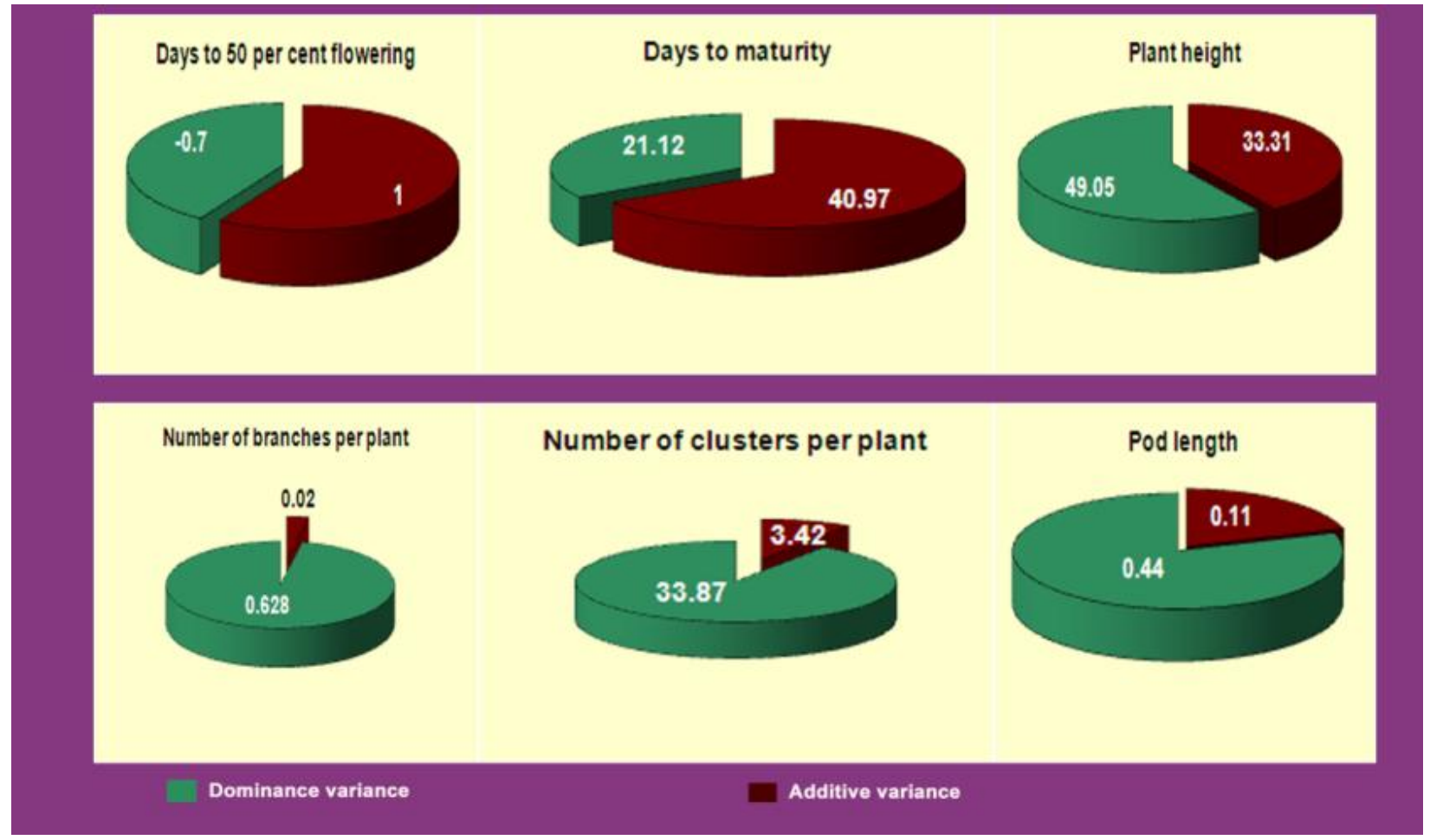

Fig.8 Magnitude of additive and dominance variance for single plant yield and its components traits

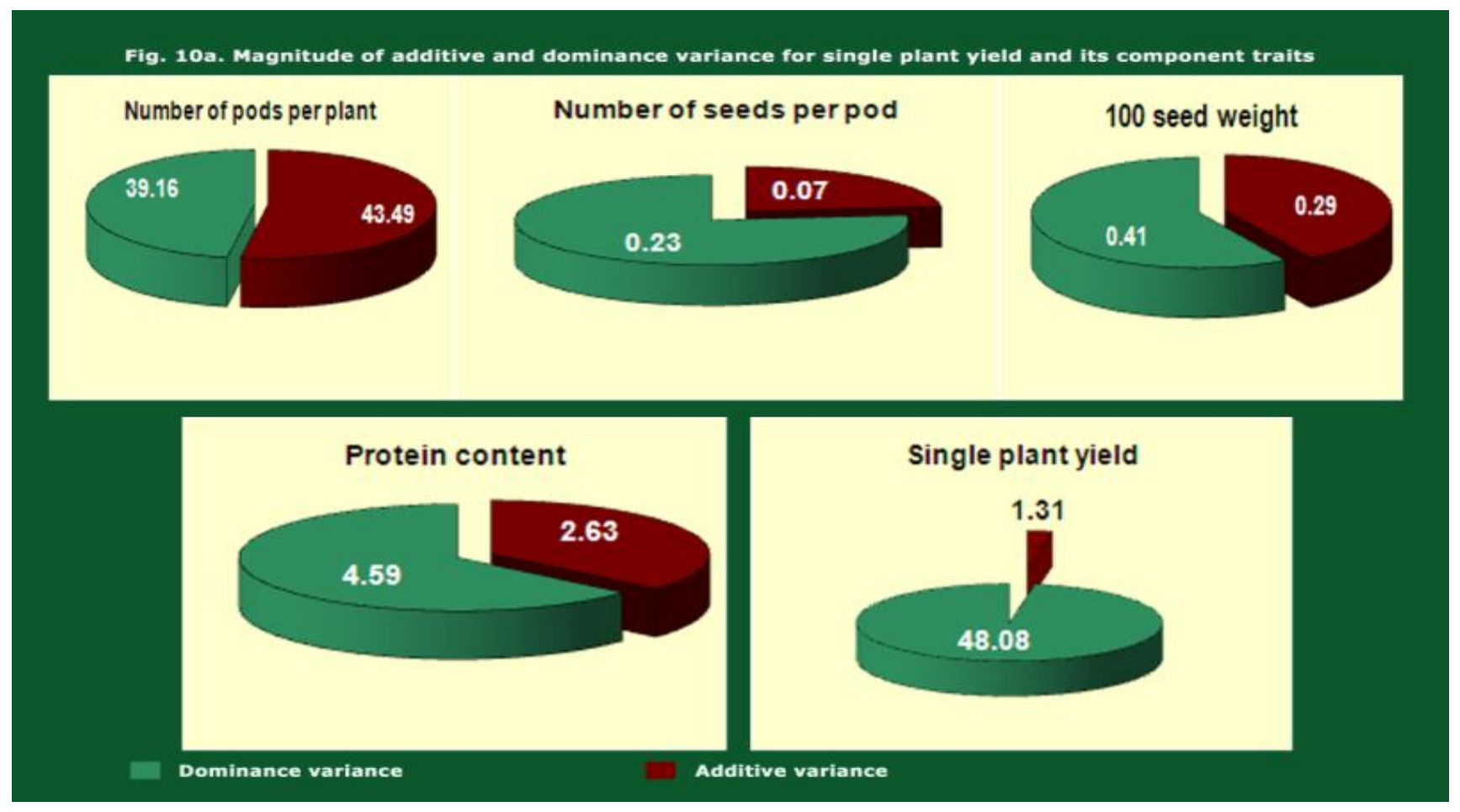


Plate.1 Hybrid selected for recombination breeding P2 X P4 (VBN 2 X CO 5)

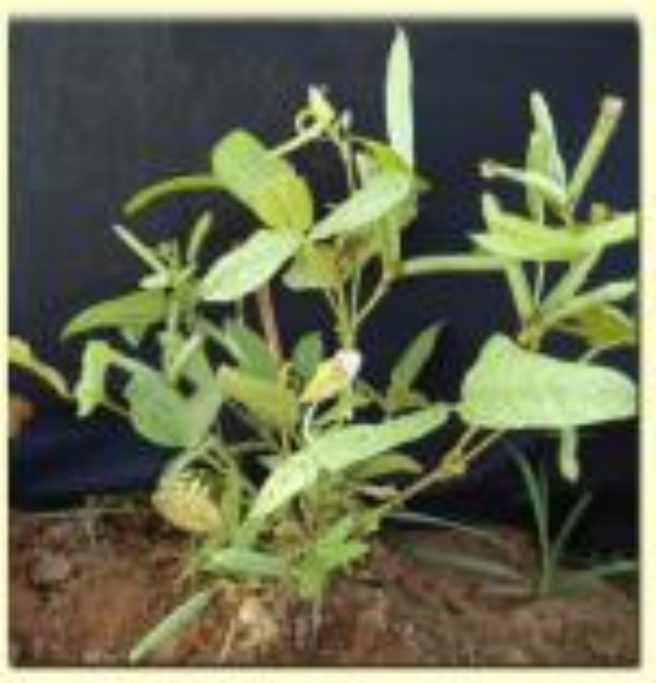

$P_{2}$

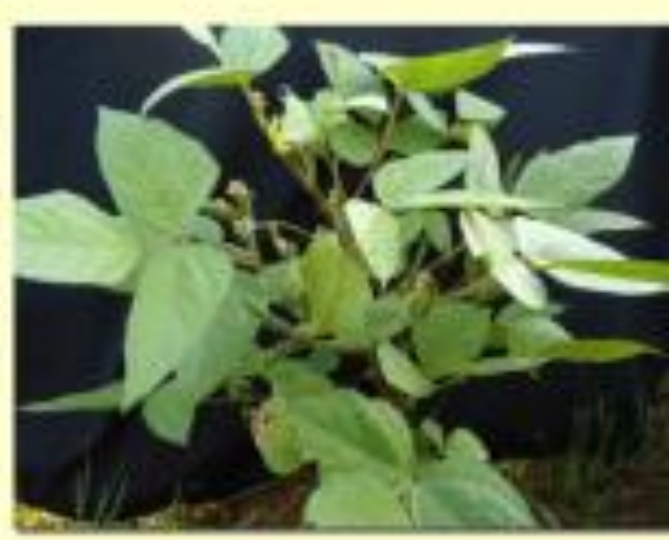

$P_{4}$

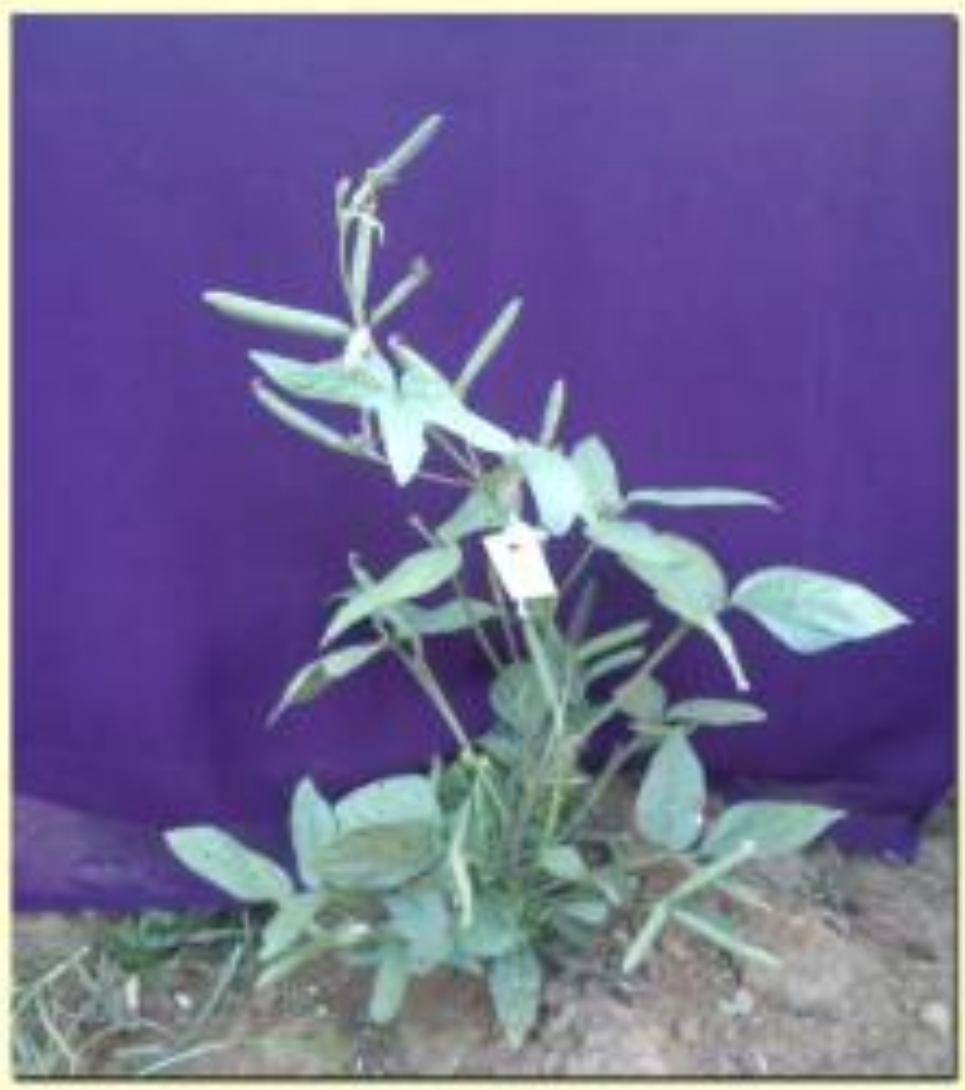

$$
P_{2} \times P_{4}
$$


Plate.2 Hybrid selected for recombination breeding P2 X P3 (VBN 2 X LBG 17)

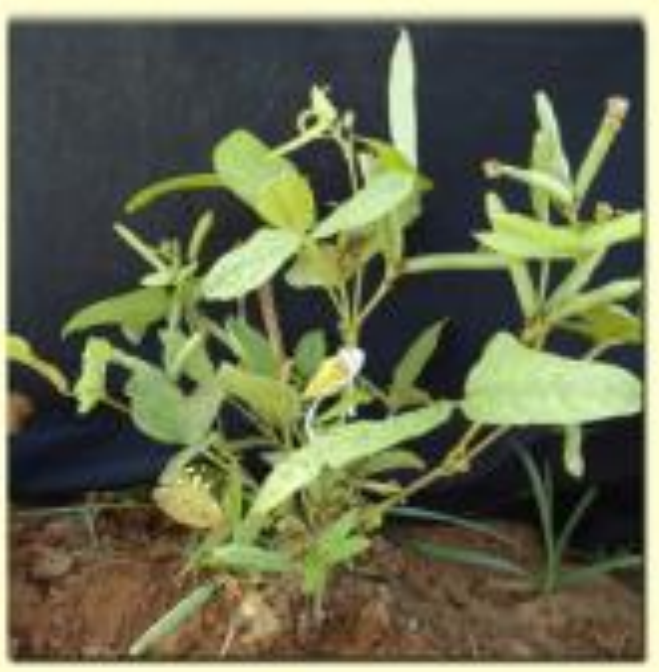

$\mathbf{P}_{2}$

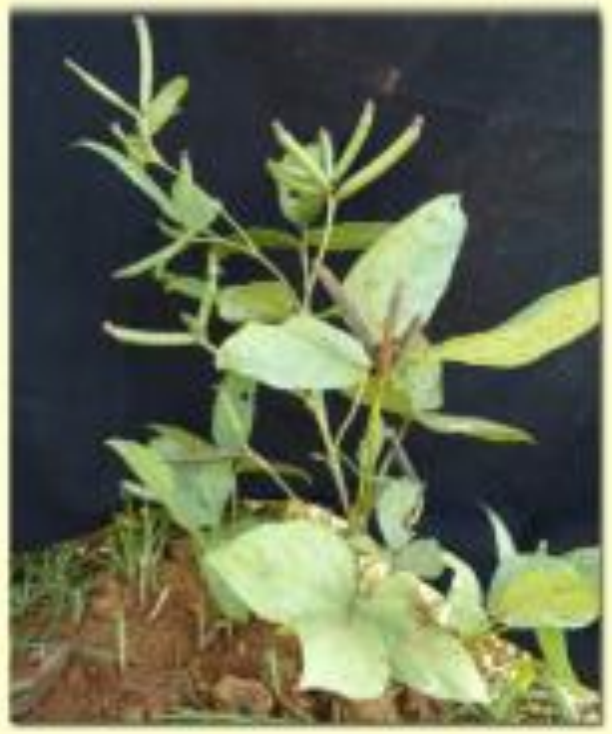

$\mathbf{P}_{3}$

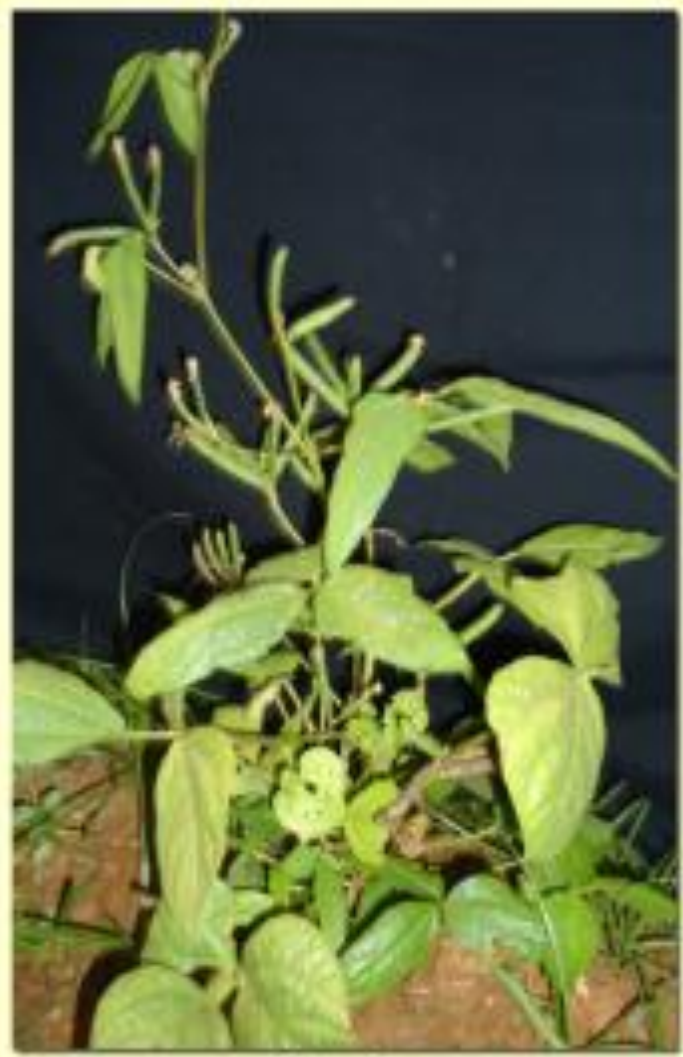

$P_{2} \times P_{3}$ 
Plate.3 Hybrid selected for recombination breeding P3 X P4 (LBG 17 X CO 5)
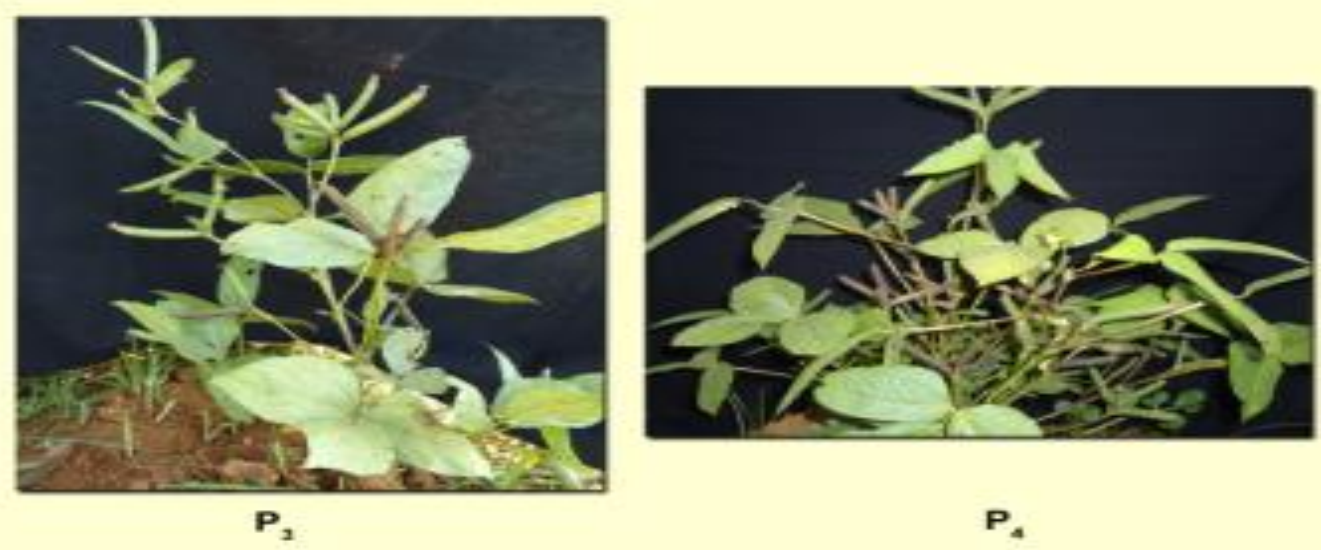

$\mathbf{P}_{\mathbf{2}}$

$\mathbf{P}_{4}$

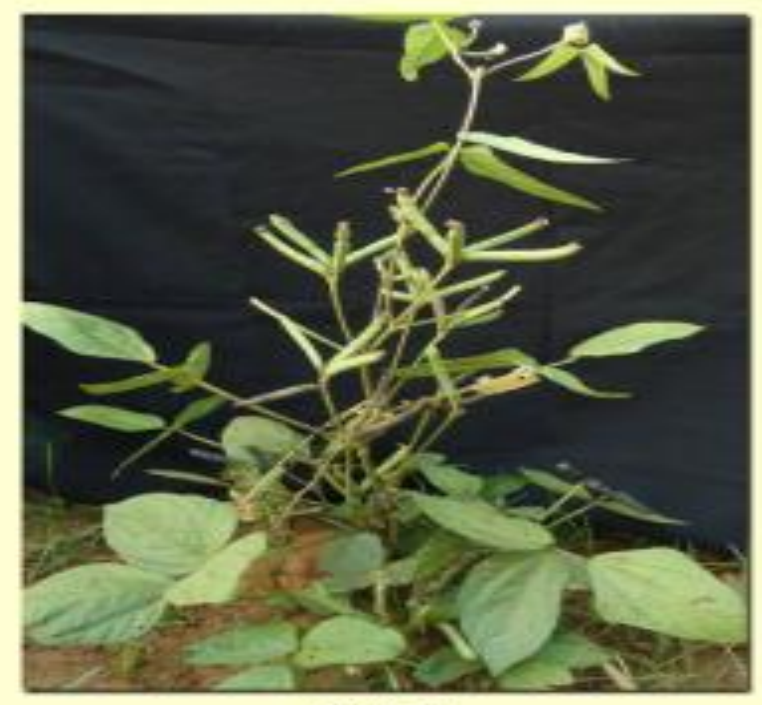

$P_{3} \times P_{4}$

The estimate of additive genetic variance (D) in combining ability analysis was more than dominant genetic variance $\left(\mathrm{H}_{1}\right)$ indicating the predominance of additive gene action for this trait. Abdul Ghaffor and Zahoor Ahmed (2005) and Yashpal et al., 2015 found additive type of gene action for number of pods per plant. The cross $\mathrm{P}_{1} \times \mathrm{P}_{4}(-9.09)$ had the lowest relative heterosis and the hybrid $\mathrm{P}_{2}$ x $\mathrm{P}_{1}$ (43.44 percent) had the highest relative heterosis for number of pods per plant. The hybrids $\mathrm{P}_{2} \times \mathrm{P}_{1}, \mathrm{P}_{3} \times \mathrm{P}_{1}, \mathrm{P}_{4} \times \mathrm{P}_{2}, \mathrm{P}_{3} \times \mathrm{P}_{2}, \mathrm{P}_{1} \times$ $\mathrm{P}_{2}, \mathrm{P}_{2} \times \mathrm{P}_{3}, \mathrm{P}_{4} \times \mathrm{P}_{1}, \mathrm{P}_{1} \times \mathrm{P}_{3}, \mathrm{P}_{3} \times \mathrm{P}_{4}, \mathrm{P}_{2} \times \mathrm{P}_{4}$ and $\mathrm{P}_{4} \times \mathrm{P}_{3}$ recorded highly significant positive relative heterosis. $\mathrm{P}_{1} \times \mathrm{P}_{4}$ (-9.09 percent) only showed highly significant negative relative heterosis. Heterobeltiosis ranged between $4.11\left(\mathrm{P}_{4} \times \mathrm{P}_{1}\right)$ to 40.48 percent $\left(\mathrm{P}_{2} \times \mathrm{P}_{1}\right)$ and with this six hybrids recorded highly positive and significant heterobeltiosis such as $\mathrm{P}_{2} \times \mathrm{P}_{1}$, $\mathrm{P}_{1} \times \mathrm{P}_{2}, \mathrm{P}_{3} \times \mathrm{P}_{1}$ and $\mathrm{P}_{3} \times \mathrm{P}_{2}$. Out of 12 hybrids, a total of three hybrids showed highly negative significant heterobeltiosis. Hybrids $\mathrm{P}_{3} \times \mathrm{P}_{4}$ and $\mathrm{P}_{4} \times \mathrm{P}_{1}$ showed significant positive heterosis over better parent. Standard heterosis ranged from $-6.69\left(\mathrm{P}_{3} \times \mathrm{P}_{2}\right)$ to 3.95 percent $\left(\mathrm{P}_{4} \times \mathrm{P}_{2}\right)$. Hybrids showing highly significant and negative heterosis over standard check were $\mathrm{P}_{1} \times \mathrm{P}_{4}, \mathrm{P}_{1} \times \mathrm{P}_{2}, \mathrm{P}_{1} \times \mathrm{P}_{3}$, $\mathrm{P}_{2} \times \mathrm{P}_{4}, \mathrm{P}_{2} \times \mathrm{P}_{3}, \mathrm{P}_{2} \times \mathrm{P}_{1}$ and $\mathrm{P}_{3} \times \mathrm{P}_{2}$ and $\mathrm{P}_{4} \times$ 
$\mathrm{P}_{2}$ and $\mathrm{P}_{3} \times \mathrm{P}_{4}$ showed positive significant heterosis (Table 6).

The range for this Number of seeds per pod varied from $6.02\left(\mathrm{P}_{2}\right)$ to $6.84\left(\mathrm{P}_{4}\right)$. The mean value for this trait was 6.07. Among the parents $\mathrm{P}_{4}$ alone showed significantly superior mean performance than the grand mean for this trait. The variation for this trait ranged from (6.19) $\mathrm{P}_{1} \times \mathrm{P}_{3}$ to (7.29) $\mathrm{P}_{3} \times \mathrm{P}_{1}$. The grand mean for this trait was (6.07). Out of 12 hybrids, $\mathrm{P}_{3} \times \mathrm{P}_{1}$ alone registered significantly more number of seeds per pod with that of the grand mean (6.62). The gca effects for number of seeds per pod ranged from $\mathrm{P}_{1}$ (0.056) to $\mathrm{P}_{4}(0.099)$ in the parents. Among the four parents all the traits showed nonsignificant $g c a$ effects. The $s c a$ effects for seeds per pod ranged from $-0.32\left(\mathrm{P}_{4} \mathrm{x}_{2}\right)$ to $0.43\left(\mathrm{P}_{2} \times \mathrm{P}_{3}\right)$. The only three crosses $\mathrm{P}_{2} \times \mathrm{P}_{3}$ alone showed significantly positive sca effects and $\mathrm{P}_{3} \times \mathrm{P}_{1}$ and $\mathrm{P}_{4} \times \mathrm{P}_{2}$ exhibited significant negative effect for this trait. The cross $\left(\mathrm{P}_{3} \times \mathrm{P}_{1}\right)$ had the highest value for all the three types in this two heterosis for the trait seeds per pod (20.93 and 20.36 percent) respectively. The estimate of dominance genetic variance $\left(\mathrm{H}_{1}\right)$ was greater than additive genetic variance (D). This showed preponderance of dominance gene action for this trait. Vaithiyalingam et al., (2002), Barad et al., (2008) and Baradhan and Thangavel (2011), Thamodharn et al., (2016) suggested the importance of non-additive gene action in determining this character. The importance of additive gene action for number of seeds per pod was confirmed by Khattak et al., (2001), Pooran Chand and Raghunadha Rao (2002), Srividhya et al., (2005), Isha Parveen et al., (2013) and Thamodharn et al., (2016). Both additive and non-additive type of gene action for determining this character was supported by Jahagirdar (2001) and Singh et al., (2007). The hybrid (14.33 percent) $\mathrm{P}_{3} \times \mathrm{P}_{2}$ showed the lowest relative heterosis and the hybrids namely $\mathrm{P}_{3} \times \mathrm{P}_{1}, \mathrm{P}_{2} \times \mathrm{P}_{3}$ and $\mathrm{P}_{3} \times \mathrm{P}_{1}$ recorded highly significant positive relative heterosis. The lowest percentage of heterobeltiosis was observed in the hybrid $\mathrm{P}_{2} \quad \mathrm{x} \quad \mathrm{P}_{3} \quad(13.45$ percent). Three hybrids recorded highly significant and positive heterobeltiosis viz., $\mathrm{P}_{3}$ x $\mathrm{P}_{1}, \mathrm{P}_{3} \times \mathrm{P}_{2}$ and $\mathrm{P}_{2} \times \mathrm{P}_{3}$ over the better parent (Table 6).

Hundred seed weight varied from $4.63\left(\mathrm{P}_{2}\right)$ to 5.91g $\left(\mathrm{P}_{4}\right)$. The parent $\mathrm{P}_{4}$ alone exhibited significantly more hundred seed weight than the grand mean $(5.07 \mathrm{~g})$. The highest value for this trait was registered by the hybrid $(6.03 \mathrm{~g}) \mathrm{P}_{4} \times \mathrm{P}_{2}$ and the lowest by the hybrid $\mathrm{P}_{3} \times \mathrm{P}_{4}(4.78 \mathrm{~g})$. Out of 12 crosses, only two hybrids showed significantly more seed weight over the grand mean value $(5.47 \mathrm{~g})$. The range for this trait was from $\mathrm{P}_{3}(-0.068)$ to $\mathrm{P}_{4}(0.236)$. Positive and significant $g c a$ effects was observed in $\mathrm{P}_{4}$. Among the parents, $\mathrm{P}_{2}, \quad \mathrm{P}_{1}$ and $\mathrm{P}_{3}$ registered nonsignificant gca effect for this trait. The minimum and maximum sca effects were recorded in $-0.22\left(\mathrm{P}_{4} \times \mathrm{P}_{2}\right)$ and $0.56\left(\mathrm{P}_{3} \times \mathrm{P}_{2}\right)$. With this trait the hybrids $\mathrm{P}_{3} \times \mathrm{P}_{2}, \mathrm{P}_{1} \times \mathrm{P}_{3}, \mathrm{P}_{3} \times$ $\mathrm{P}_{1}$ and $\mathrm{P}_{2} \times \mathrm{P}_{4}$ recorded significant and positive $s c a$ effects and the cross $\mathrm{P}_{4} \times \mathrm{P}_{3}, \mathrm{P}_{2} \mathrm{X}$ $\mathrm{P}_{1}, \mathrm{P}_{4} \times \mathrm{P}_{1}$ and $\mathrm{P}_{4} \times \mathrm{P}_{2}$ showed significant and negative sca effects for this trait. The heterosis percentage over mid parent varied from - $12.31\left(\mathrm{P}_{3} \times \mathrm{P}_{4}\right)$ to 19.73 percent $\left(\mathrm{P}_{1} \mathrm{x}\right.$ $\mathrm{P}_{3}$ ). The combining ability analysis clearly revealed the major role of non-additive gene action governing this character since $\mathrm{H}_{1}$ was higher than D. Govindaraj and Subramanian (2001), Vaithiyalingam et al., (2002), Pooran Chand Jayapradha et al., (2005), Thangavel (2011), Bhagirathram et al., (2013) and Tantasawat et al., (2015) were of the opinion that 100 seed weight was controlled by nonadditive gene action. However, Indrani Dana and Das Gupta (2001), Abdul Ghaffor and Zahoor Ahmed (2005) and Saif Ullah Ajmal et al., (2007) indicated that this character was controlled by additive gene action.Five hybrids viz., $\mathrm{P}_{1} \times \mathrm{P}_{3}, \mathrm{P}_{2} \times \mathrm{P}_{1}, \mathrm{P}_{3} \times \mathrm{P}_{2}$ and $\mathrm{P}_{4} \mathrm{x}$ 
$\mathrm{P}_{2}$ recorded highly significant and positive relative heterosis and (-12.31 percent) $\mathrm{P}_{3} \times \mathrm{P}_{4}$ alone showed highly significant and negative relative heterosis. Heterobeltiosis ranged between -13.59 ( $\left.\mathrm{P}_{1} \times \mathrm{P}_{4}\right)$ and 19.13 percent $\left(\mathrm{P}_{1} \times \mathrm{P}_{3}\right)$ with four hybrids this two hybrids $\mathrm{P}_{3}$ $x \mathrm{P}_{4}$ and $\mathrm{P}_{1} \times \mathrm{P}_{4}$ showed highly significant and negative heterobeltiosis over better parent. The standard heterosis over a standard variety ranged between $-13.59\left(\mathrm{P}_{1} \times \mathrm{P}_{4}\right)$ and $10.72\left(\mathrm{P}_{3}\right.$ x $\mathrm{P}_{1}$ ). The hybrids namely $\mathrm{P}_{3} \times \mathrm{P}_{4}, \mathrm{P}_{1} \times \mathrm{P}_{2}, \mathrm{P}_{2}$ $x \mathrm{P}_{3}$ and $\mathrm{P}_{1} \times \mathrm{P}_{4}$ recorded highly significant and negative standard heterosis. Hybrid $\mathrm{P}_{3} \mathrm{X}$ $\mathrm{P}_{1}$ recorded significant positive standard heterosis (Table 7).

The range for protein content was from 16.17 $\left(\mathrm{P}_{2}\right)$ to 20.05 percent $\left(\mathrm{P}_{1}\right)$. The grand mean for this trait was 18.32 percent. The parent $\mathrm{P}_{1}$ was showed the maximum protein content followed by $\mathrm{P}_{4}$ and $\mathrm{P}_{2}$ recorded significantly positive mean performance than the grand mean for this trait. Protein content showed a wide range, from (17.14) $\mathrm{P}_{1} \times \mathrm{P}_{3}$ to $(21.15$ percent) $\mathrm{P}_{4} \times \mathrm{P}_{2}$. Out of 12 hybrids, seven hybrids exhibited significantly superior mean values than the grand mean (20.26 percent). Protein content of parents varied from $\mathrm{P}_{3}$ ($0.216)$ to $\mathrm{P}_{4}(0.554)$ (Table 11). Positive and significant gca effect was registered only in $\mathrm{P}_{4}$ and other two parents $\mathrm{P}_{2}$ and $\mathrm{P}_{3}$ exhibited negatively significant gca effects for this trait. The hybrids had the lowest and the highest sca effects of $-0.54\left(\mathrm{P}_{4} \times \mathrm{P}_{3}\right)$ and $1.44\left(\mathrm{P}_{4} \mathrm{X}\right.$ $\mathrm{P}_{1}$ ). Hybrides $\mathrm{P}_{4} \times \mathrm{P}_{1}, \mathrm{P}_{2} \times \mathrm{P}_{4}, \mathrm{P}_{2} \times \mathrm{P}_{3}, \mathrm{P}_{2} \times \mathrm{P}_{1}$ and $\mathrm{P}_{3} \times \mathrm{P}_{4}$ showed significant and positive sca effects. Out of 12 hybrids, a total of five crosses registered significant and negative sca effects. The $\mathrm{H}_{1}$ value was higher than $\mathrm{D}$. This inferred the role of non-additive gene action for this trait. Anbumalarmathi et al., (2004) and Barad et al., (2008) also observed predominance of non-additive gene action in controlling this trait. Aher et al., (2001) and Pooran Chand and Raghunadha Rao (2002) suggested that the character was controlled by additive type of gene action. The relative heterosis for this trait ranged from-9.09 $\left(\mathrm{P}_{1} \mathrm{X}\right.$ $\left.\mathrm{P}_{3}\right)$ to 20.88 percent $\left(\mathrm{P}_{3} \times \mathrm{P}_{2}\right)$ with five hybrids $\mathrm{P}_{3} \times \mathrm{P}_{2}, \mathrm{P}_{4} \times \mathrm{P}_{2}, \mathrm{P}_{2} \times \mathrm{P}_{4}, \mathrm{P}_{4} \times \mathrm{P}_{3}$ and $\mathrm{P}_{3}$ $x P_{1}$ recorded highly significant and positive relative heterosis and the hybrids $\mathrm{P}_{4} \times \mathrm{P}_{1}$ and $\mathrm{P}_{1} \times \mathrm{P}_{3}$ recorded highly significant negative heterosis over mid parent. The lowest and highest value for better parent heterosis was shown by the hybrids $\mathrm{P}_{1} \times \mathrm{P}_{2}(-6.53)$ and $\mathrm{P}_{3} \mathrm{X}$ $\mathrm{P}_{2}$ (14.76 percent) respectively and the crosses which showed highly significant and positive heterosis were as follows $\mathrm{P}_{3} \times \mathrm{P}_{2}, \mathrm{P}_{4}$ $\times \mathrm{P}_{2}$ and $\mathrm{P}_{4} \times \mathrm{P}_{3}$ and four hybrids registered highly significant and negative heterosis over better parent. Standard heterosis varied between -4.97 $\left(\mathrm{P}_{3} \times \mathrm{P}_{4}\right)$ and 5.48 percent $\left(\mathrm{P}_{4} \mathrm{X}\right.$ $\mathrm{P}_{2}$ ) and six hybrids showed highly significant and positive heterosis, $\mathrm{P}_{3} \times \mathrm{P}_{4}$ alone recorded significantly negative heterosis and one cross $\mathrm{P}_{4} \times \mathrm{P}_{2}$ recorded highly significant positive heterosis over the check variety (Table 7).

The trait single plant yield ranged from 7.13 $\left(\mathrm{P}_{2}\right)$ to $10.06 \mathrm{~g}\left(\mathrm{P}_{4}\right)$. The mean value for this trait was $8.69 \mathrm{~g}$. The parents $\mathrm{P}_{2}$ and $\mathrm{P}_{4}$ registered significantly higher seed yield to their mean (Fig. 3). This trait also showed wide a range, which was from $8.29\left(\mathrm{P}_{1} \times \mathrm{P}_{2}\right)$ to $20.69 \mathrm{~g}\left(\mathrm{P}_{4} \times \mathrm{P}_{3}\right)$ and closely followed by the cross $\mathrm{P}_{3} \times \mathrm{P}_{4}$. Among the 12 hybrids studied, 11 hybrids showed significantly superior mean performance when compared to grand mean $(14.19 \mathrm{~g})$. The parent $\mathrm{P}_{1}$ had the lowest value of $(-0.702), \mathrm{P}_{4}$ while exhibited the highest value of (1.997) for the gca effects. The parents $\mathrm{P}_{4}$ and $\mathrm{P}_{3}$ had significant and positive gca effects, and the parents $\mathrm{P}_{2}$ and $\mathrm{P}_{1}$ recorded significant and negative $\mathrm{gca}$ effects for this trait. The sca effects for yield ranged from $-0.38\left(\mathrm{P}_{4} \times \mathrm{P}_{2}\right)$ to $3.63\left(\mathrm{P}_{3} \times \mathrm{P}_{4}\right)$. Five hybrids recorded significant and positive $s c a$ effects and the six hybrids recorded negative and significant $s c a$ effects for single plant yield. This was supported by the earlier reports of Govindaraj and Subramanian 
(2001), Pooran Chand and Raghunadha Rao (2002), Vaithiyalingam et al., (2002), Anbumalamathi et al., (2004), Abdul Ghaffor and Zahoor Ahmad (2005), Kachave et al., (2015) and Tnamodharn et al., (2016), whereas the importance of additive gene action for seed yield was suggested by Aher et al., (2001), Abdul Ghaffor and Zahoor Ahmad (2003) and Srividhya et al., (2005). Khattak et al., (2001), Indrani Dana and Das Gupta (2001), Singh et al., (2007) and Yashpal et al., (2015) reported both additive and non-additive type gene action for single plant yield. The cross $\left(\mathrm{P}_{1} \times \mathrm{P}_{3}\right)$ showed the lowest value of heterosis for all the three traits this two for yield (-7.85 and -17.56 percent). Simultaneously hybrid $\left(\mathrm{P}_{4} \times \mathrm{P}_{3}\right)$ recorded the maximum heterosis value for all the three bases (121.71, 106.97 and 105.73 percent). 11 crosses recorded highly significant and positive relative heterosis over mid parent. The crosses which showed highly significant and positive heterobeltiosis were $\mathrm{P}_{4} \times \mathrm{P}_{3}, \mathrm{P}_{3} \times$ $\mathrm{P}_{4}, \mathrm{P}_{4} \times \mathrm{P}_{1}, \mathrm{P}_{3} \times \mathrm{P}_{1}, \mathrm{P}_{3} \times \mathrm{P}_{2}, \mathrm{P}_{4} \times \mathrm{P}_{2}, \mathrm{P}_{1} \times \mathrm{P}_{4}, \mathrm{P}_{2}$ $\times \mathrm{P}_{1}, \mathrm{P}_{2} \times \mathrm{P}_{4}, \mathrm{P}_{1} \times \mathrm{P}_{3}$ and $\mathrm{P}_{2} \times \mathrm{P}_{3}$. Hybrids $\mathrm{P}_{1} \times$ $\mathrm{P}_{2}$ recorded highly significant negative heterobeltiosis. $\mathrm{P}_{4} \times \mathrm{P}_{3}, \mathrm{P}_{3} \times \mathrm{P}_{4}, \mathrm{P}_{4} \times \mathrm{P}_{1}, \mathrm{P}_{3} \times$ $\mathrm{P}_{1}, \mathrm{P}_{1} \times \mathrm{P}_{4}, \mathrm{P}_{4} \times \mathrm{P}_{2}, \mathrm{P}_{2} \times \mathrm{P}_{4}, \mathrm{P}_{3} \times \mathrm{P}_{2}, \mathrm{P}_{2} \times \mathrm{P}_{1}$ and $\mathrm{P}_{1} \times \mathrm{P}_{3}$ were the hybrids showing highly significant and positive standard heterosis over the standard variety and hybrids $\mathrm{P}_{1} \mathrm{x} \mathrm{P}_{2}$ exhibited highly significant and negative standard heterosis. High value of dominance genetic variance $\left(\mathrm{H}_{1}\right)$ than the additive variance (D) indicated that the character was determined by non-additive gene action (Table 7) (Figs. 7, 8).

Among the hybrids, LBG 17 x CO 5 recorded significant standard heterosis for 10 traits except for number of seeds per plant. The hybrid VBN 2 x LBG 17 recorded desirable standard heterosis for eight traits except number of branches per plant and single plant yield and VBN 2 x CO 5 showed desirable standard heterosis for eight traits except 100 grain weight and protein content (Table 8 and 9). It was followed by VBN 4x VBN 2 and VBN 4 x LBG 17 which showed significant standard heterosis for eight traits except number of branches per plant, pod length and 100 grain weight (Plate 1, 2 \& 3). Similar results were also reported by Jiji Joseph and Santhoshkumar (2000) for plant height and number of branches per plant, Loganathan et al., (2001) and Barad et al., (2008) for number of seeds per pod (Table 10).

The phenotypic coefficient of variation ranged from 2.84 to 30.07 per cent. Higher percent of PCV was recorded by single plant yield followed by, number of cluster per plant, number of branches per plant, plant height, number of pods per plant and 100 grain weight (Table 4) (Fig. 1). The lowest value of PCV was recorded by days to 50 percent flowering. The genotypic coefficient of variation ranged from 2.15 to 29.90 per cent. Higher per cent of GCV was recorded by single plant yield followed by number of clusters per plant, plant height, number of pods per plant and number of branches per plant (Fig. 2). The lowest value of GCV was recorded by days to 50 percent flowering. The heritability ranged from 31.54 to 99.30 per cent. The highest value of heritability was recorded in plant height followed by single plant yield, number of cluster per plant, number of pods per plant, days to maturity and protein content. The lowest percentage of heritability was recorded by number of seeds per pod. The genetic advance varied from 3.35 to 61.26 per cent. The highest value of genetic advance was observed in single plant yield followed by number of cluster per plant, plant height, number of pod per plant and number of branches per plant. The lowest value of genetic advance was recorded in days to 50 per cent flowering.

Among the 12 crosses, three hybrids showed complete resistance against YMV with high 
yield performance. The hybrids are VBN $4 \mathrm{x}$ VBN 2, VBN 2 x VBN 4 and VBN 2 x LBG 17. So the segregants from these crosses may be utilized for recombination breeding for hybridization and YMV resistant (Table 13). Four hybrids reacted as moderately resistant to YMV such as VBN $4 \times$ LBG 17, VBN $2 x$ CO 5, LBG 17 x VBN 4 and CO 5 x VBN 2. Moderately susceptible reactions recorded by the hybrids were VBN 4 x CO5, LBG $17 \mathrm{x}$ $\mathrm{CO} 5$ and $\mathrm{CO} 5 \times \mathrm{VBN} 4$. The hybrids viz., LBG $17 \times$ VBN 2 and CO 5 x LBG 17 was the two crosses showing susceptible reaction against YMV. Similar results were also reported by Shamim et al., (2014) and Peeta et al., (2016). From the foregoing discussion it may be concluded that the pod length and number of branches per plant expressed dominant gene action followed by number of pods per plant additive gene action found to be best selection criteria for yield improvement in blackgram as revealed by path analysis. Similar result was reported by Prasanthi et al., (2013). The parents for general combiners and the cross involving the same will expect through useful segregants also the study revealed that the hybrid LBG $17 \times$ CO 5 and VBN 2 x LBG 17 found to be superior which combiners yield and quality characters and this hybrids can be exploited for heterosis breeding. Among the above hybrids VBN 2 x LBG 17 which is having the parents resistance for YMV and powdery mildew respectively, expect through more yield combined with resistance and can be commercially exploited.

\section{Yellow Mosaic Virus disease (YMV)}

The Yellow Mosaic Virus Disease (YMV) incidence was recorded on all the plants based on the visual scores on $50^{\text {th }}$ day while the susceptible check $\mathrm{C} 05$ recorded scale 6.9. The classification was made into scales $1-9$ as follows based on the scale adopted by Singh et al., (1988).

\section{References}

Abdul Ghafoor and Zahoor Ahmad. 2003. Exploitation of (Vigna mungo (L.) Hepper) germplasm using multivariate analysis based on agronomic traits. Pak. J. Bot., 35(2): 187-196.

Abdul Ghafoor and Zahoor Ahmad. 2005. Diversity of agronomic traits and total seed protein in blackgram (Vigna mungo (L.) Hepper). Acta Biologica Cracoviensia Series Botanica, 47(2): 69-75.

Aher, R.P., D.V. Dahat and P.P. Surve. 2001. Diallel analysis for yield contributing characters in mungbean. Legume Res., 24(2): 124-126.

Anbumalarmathi, J., P. Rangasamy and S. Babu. 2004. Combining ability and heterosis for yield and yield components in greengram (Vigna radiata (L.) Wilczek). Madras Agric. J., 91(1-3): 79-82.

Baker, R.G. 1978. Issues in diallel analysis. Crop Sci., 18: 533-536.

Barad, H.R., M.S. Pithia and J.H. Vachhani. 2008. Heterosis and combining ability studies for economic traits in genetically diverse lines of mungbean (Vigna radiata (L.) Wilczek). Legume Res., 32(1): 68-71.

Barad, H.R., M.S. Pithia and J.H. Vachhani. 2008. Heterosis and combining ability studies for economic traits in genetically diverse lines of mungbean (Vigna radiata (L.) Wilczek). Legume Res., 32(1): 68-71.

Baradhan, G. and P. Thangavel. 2011. Gene action and combining ability for yield and other quantitative traits in blackgram (Vigna mungo (L) Hepper). Plant Archives, 11(1): 267-270.

Bhagirath Ram, SBS., Tikka and S Acharya. 2013 Heterosis and combining ability in blackgram (Vigna mungo) under different environments. Indian $\mathrm{J}$. of 
Agricultural Sciences, 83(6): 611-6.

Bos, L. 1999. Plant Viruses: Unique and Intriguing Pathogens: A Text Book of Plant Virology, Backhuys Publishers, the Netherlands. pp: 305-306.

Dhillon, B.S. 1975. The application of partial diallel crosses in plant breeding. Crop Improv., 2: 1 -7.

Govindaraj, P. and M. Subramanian. 2001. Combining ability analysis in blackgram (Vigna mungo (L.) Hepper). Madras Agric. J., 88(4-6): 237-240.

Hull, R. 2004. Mathew's Plant Virology, Fourth Edition. Elsevier Publishers, India. pp: 180-182.

Indirani Dana and T. Dasgupta. 2001. Combining ability in blackgram. Indian J. Genet., 61(2): 170-171.

Isha Parveen S., M Reddi Sekhar, DM Reddy and P Sudhakar. 2013. Heterosis in Blackgram (Vigna mungo L. Hepper) History and Future Thrust. Research and Reviews: Journal of Agriculture and Allied sciences. 2(4): 21-25.

Jahagirdar, J.E. 2001. Heterosis and combining ability studies for seed yield and yield components in mungbean. Indian J. Pulse Res., 14(2): 141-142.

Jayaprada, M., K. Raja Reddy, M. Reddy Sekhar and G. Lakshmikanta Reddy. 2005. Genetic analysis of quantitative characters in mungbean (Vigna radiata (L.) Wilczek). Legume Res., 28(3): 210-215.

Jiji Joseph and A.V. Santhoshkumar. 2000. Genetic analysis of metric traits in greengram (Vigna radiata (L.) Wilczek). Inter. J. Trop. Agric., 18(2): 133-139.

Kachave G.A., Parde N.S., Zate D.K. and Harer P.N. 2015. Analysis of combining ability in Blackgram (Vigna mungo L.Hepper). Inter. J. of Advanced Research. 3(3): 1139-1146.

Khattak, G.S.S., M.A. Haq, Muhammad Ashraf and G.R. Tahir. 2001. Genetic basis of synchrony in pod maturity in mungbean (Vigna radiata (L.) Wilczek). Kasetsart J. (Nat. Sci.). 35: 17.

Loganathan, P., K. Saravanan, P. Thangavel and J. Ganesan. 2001. Heterosis for yield and yield components in greengram (Vigna radiata (L.) Wilczek). Legume Res., 24(2): 77-81

Manivannan, N. 2002. Association analysis in segregating generations of greengram (Vigna radiate (L.) Wilczek). Legume Res., 25(1): 63-65.

Panse, V.G. 1942. Genetics of quantitative characters in relation to plant breeding. Indian J. Genet., 2: 318-327.

Peeta Gopi, Satyanarayana A, Rama Krishna A and Sambasiva Rao KRS. 2016. Evaluation of Blackgram Germplasm for Resistance against YMV. J Plant Pathol Microbiol. 7(7): 21577471.1000368.

Pooran Chand and C. Raghunadha Rao. 2002. Studies on gene action in a biparental cross in blackgram (Vigna mungo (L.) Hepper). Indian J. Genet., 62(4): 347348.

Prasanthi L. Reedy B.V Bhaskara, Geetha B, Jothi Ramya, Abhishek. 2013. Molecular marker for screening Yellow Mosaic disease resistance in (Vigna mungo L. Hepper). Ele. J. of Plant Breeding, 4(2): 1137-1141.

Saif Ullah Ajmal, Muhammad Zubair and Muhammad Anwar. 2007. Genetic implication of yield and its components in mungbean (Vigna radiata (L.) Wilczek). Pak. J. Bot., 39(4): 12291236.

Shamin M.Z and Pandey A. 2016. Identification of Yellow Mosaic Virus (YMV) resistant Blackgram (Vigna mungo L. Hepper) genotypes for cultivation in Northern India. Journal of Agroecology and Natural Resource Management. 1(2): 48-50. 
Shull, G.H. 1914. Duplicate genes for capsule form in Bursa bursapastoris. Z. Ind. Abstr. Ver., 12: 97-149.

Singh, B.B. and H.K. Dikshit. 2003. Combining ability studies for yield and architechtural traits in mungbean (Vigna radiata (L.) Wilczek). Indian J. Genet., 63(4): 351-352.

Singh, B.R., M. Singh, M.D. Yadav and S.M. Dinger. 1982. Yield loss in mungbean due to yellow mosaic. Sci. Cult., 48: 435-436.

Singh, G., S. Kapoor and K. Singh. 1988. Multiple disease resistance in mungbean with special emphasis on mungbean yellow mosaic virus. In: International symposium on mungbean, 2nd Nov.1620, Bangkok, Tailand, pp: 290-296.

Singh, V.K., K. Tyagi, A.K. Tamer, M. N. Singh and R. Nandanl. 2007. Gene action for yield and yield attributing traits in mungbean (Vigna radiata (L.) Wilczek). Legume Res., 30(1): 29-32.

Srividhya, A., M. Reddy Sekhar, G.L.K. Reddy and K.S. Reddy. 2005. Components of genetic variation in biparental progenies of blackgram (Vigna mungo (L.) hepper). Legume Res., 28 (4): 291 - 293.

Supriyo Chakraborty., H.K. Borah, B.K. Borah, Dalim Pathak, B.K. Baruah, Hemen Kalita, and Bhubaneswar Barman. 2010. Genetic Parameters and Combining Ability Effects of Parents for Seed Yield and other Quantitative
Traits in Black Gram (Vigna mungo (L.) Hepper). Not Sci Biol., 2 (2): 121-126.

Tantasawat P.A., P. Khajudparn, T. Prajongjai and O. Poolsawat. 2015. Heterosis for the improvement of yield in mungbean (Vigna radiata (L.) Wilczek). Genet. Mol. Res. 14 (3): 10444-10451.

Thamodharan G, Geetha S. and R. Ushakumari. 2016. Studies on heterosis in blackgram (Vigna mungo (L.) Hepper). Indian J. Agric. Res., 50 (5): 406-413.

Vaithiyalingam, M., S. Chidambaram, P. Vivekanandan and C. Vanniarajan. 2002. Combining ability studies in blackgram (Vigna mungo (L.) Hepper). Crop Res., 24(1): 81-85.

Vijay Kumar G, M Vanaja, P Raghu Ram Reddy, K Salini, Babu Abraham, and N Jyothi Lakshmi. 2014. Studies on Combining Ability and Genetic Advance in Blackgram (Vigna mungo). Research and Reviews: Journal of Agriculture and Allied sciences. 3(3): 14-24.

Yashpal, M. N. Singh, N. Pathak and S. K. Saroj. 2015. Combining ability, heterosis and inbreeding depression in inter specific hybrids involving greengram (Vigna radiata $(\mathrm{L}$.$) Wilczek)$ and blackgram (Vigna mungo (L.) Hepper). Ele. J. of Plant Breeding, 6(1): 87-92.

\section{How to cite this article:}

Suguna, R., P. Savitha and Ananda Kumar, C.R. 2017. Inheritance of Genetic Variability, Combining Ability and Heterosis for Yellow Mosaic Virus Disease Resistance and Yield Improvement in Blackgram [Vigna mungo (L.) Hepper]. Int.J.Curr.Microbiol.App.Sci. 6(11): 2416-2442. doi: https://doi.org/10.20546/ijcmas.2017.611.286 